\title{
Depletion of major pathogenic cells in asthma by targeting CRTh2
}

Tao Huang, ${ }^{1}$ Meredith Hazen, ${ }^{2}$ Yonglei Shang, ${ }^{2}$ Meijuan Zhou, ${ }^{1}$ Xiumin Wu, ${ }^{1}$ Donghong Yan, Zhonghua Lin, ${ }^{1}$ Margaret Solon, ${ }^{3}$ Elizabeth Luis, ${ }^{4}$ Hai Ngu, ${ }^{3}$ Yongchang Shi, ${ }^{5}$ Arna Katewa, ${ }^{1}$ David F. Choy, ${ }^{6}$ Nandhini Ramamoorthi, ${ }^{6}$ Erick R. Castellanos, ${ }^{4}$ Mercedesz Balazs, ${ }^{1}$ Min Xu, Wyne P. Lee, ${ }^{1}$ Marissa L. Matsumoto, ${ }^{4}$ Jian Payandeh, ${ }^{7}$ Joseph R. Arron, ${ }^{6}$ Jo-Anne Hongo, ${ }^{2}$ Jianyong Wang, ${ }^{5}$ Isidro Hötzel, ${ }^{2}$ Cary D. Austin, ${ }^{3}$ and Karin Reif ${ }^{1}$

Department of Immunology, ${ }^{2}$ Department of Antibody Engineering, ${ }^{3}$ Department of Pathology, ${ }^{4}$ Department of Protein Chemistry, ${ }^{5}$ Department of Biochemical and Cellular Pharmacology, ${ }^{6}$ Department of Biomarker Discovery OMNI, ${ }^{7}$ Department of Protein Engineering, Genentech Inc., South San Francisco, California, USA.

Eosinophilic inflammation and Th2 cytokine production are central to the pathogenesis of asthma. Agents that target either eosinophils or single Th2 cytokines have shown benefits in subsets of biomarker-positive patients. More broadly effective treatment or disease-modifying effects may be achieved by eliminating more than one inflammatory stimulator. Here we present a strategy to concomitantly deplete Th2 T cells, eosinophils, basophils, and type-2 innate lymphoid cells (ILC2s) by generating monoclonal antibodies with enhanced effector function (19A2) that target CRTh2 present on all 4 cell types. Using human CRTh2 (hCRTh2) transgenic mice that mimic the expression pattern of hCRTh2 on innate immune cells but not Th2 cells, we demonstrate that anti-hCRTh2 antibodies specifically eliminate hCRTh2+ basophils, eosinophils, and ILC2s from lung and lymphoid organs in models of asthma and Nippostrongylus brasiliensis infection. Innate cell depletion was accompanied by a decrease of several Th2 cytokines and chemokines. hCRTh2-specific antibodies were also active on human Th2 cells in vivo in a human Th2-PBMC-SCID mouse model. We developed humanized hCRTh2-specific antibodies that potently induce antibody-dependent cell cytotoxicity (ADCC) of primary human eosinophils and basophils and replicated the in vivo depletion capacity of their murine parent. Therefore, depletion of hCRTh2+ basophils, eosinophils, ILC2, and Th2 cells with h19A2 hCRTh2-specific antibodies may be a novel and more efficacious treatment for asthma.

Conflict of interest: All authors are or were employed by Genentech Inc. at the time of the study and hold equity in the Roche Group. In addition, the research in this manuscript was fully funded by Genentech Inc.

Submitted: January 25, 2016 Accepted: April 12, 2016 Published: May 19, 2016

Reference information: JCI Insight. 2016;1(7):e86689. doi:10.1172/jci.insight.86689.

\section{Introduction}

Asthma is a multifactorial chronic inflammatory disease of the airways. While asthma is a complex heterogeneous disease, the prevalent pathogenic mechanisms involve allergic type- 2 immune responses. Chief players in type- 2 inflammation are CD4 ${ }^{+} \mathrm{Th} 2$ cells that secrete IL4, IL5, and IL13, but also chemokines and other mediators, leading to recruitment of inflammatory leucocytes and establishment of type-2 inflammation with its hallmarks of IgE antibody production and eosinophilia. In addition to their central role in acute inflammation, Th2 memory cells that reside in the lung during disease remission contribute to the persistence and progression of asthma (1-3). Airway inflammation can also be propagated by several innate immune cells, including eosinophils, mast cells, basophils, and type-2 innate lymphoid cells (ILC2s), which can serve as alternate sources of $\mathrm{Th} 2$ cytokines and an array of other inflammatory mediators such as amphiregulin, TNFA, or GMCSF. Together, these cytokines and other mediators can promote airway remodeling, hyperreactivity, and further cellular inflammation $(4,5)$. Differences in cytokine-driven inflammation or altered innate immune cell activation triggered by a range of environmental stress factors or infectious pathogens may underlie the heterogeneity and complexity of clinical asthma $(6,7)$

Recent clinical trials in patients with uncontrolled asthma refractory to inhaled corticosteroids have revealed that blocking IL4/IL13 pathway activity or reducing eosinophil recruitment via IL5/IL5RA blockade is only efficacious in a subset of patients $(6,8)$. Consequently, type-2 biomarkers including serum periostin levels, FENO levels, sputum IL13 levels, and sputum or blood eosinophil counts are required to distinguish responders from nonresponders, resulting in a dichotomous categorization of clinical asthma into a disease with evidence of predominant type-2 inflammation (Th2-high asthma) or a disease with min- 
imal type-2 pathway activity (Th2-low asthma) $(6,9)$. While Th2-high asthma patients are characterized by high IL4/IL13 activity and/or eosinophilia, the Th2-low asthma group does not demonstrate dominant molecular phenotypes, lacks specific biomarkers, and is clinically heterogeneous, although it has been associated in at least some subgroups with neutrophilia and neutrophilic cytokines such as IL17, TNFA, and IL8. Despite the relative success of Th2 cytokine-directed therapies in reducing asthma exacerbations and function measurements in moderate-to-severe diagnostic-positive Th2-high asthma patients, evidence is emerging that these single agent therapies do not eliminate exacerbations or completely suppress other outcomes of poor asthma control even in responders $(10,11)$. Furthermore, it is currently not established that these prospective therapies will produce disease-modifying effects. Therefore, more pronounced efficacy in a larger patient population and, in particular, prolonged effects may require concomitant targeting of several key cytokine pathways or immune cells (10-12).

The chemoattractant receptor-homologous molecule expressed on Th2 cells (CRTh2), also designated CD294 or GPR44 and its gene PTGDR2, is a high-affinity prostaglandin D2 receptor that is expressed on major human cell types that mediate asthma pathogenesis, namely Th2 cells, ILC2, eosinophils, and basophils (13-16). Thus, CRTh2 ${ }^{+} \mathrm{CD}^{+} \mathrm{T}$ cells are increased in bronchoalveolar lavage (BAL) of asthma patients (17). Basophils are highly enriched in postmortem lung of fatal asthma patients, as well as in bronchial biopsies of asthmatics (18). Augmented numbers of CRTh2 ${ }^{+}$ILC2 and eosinophils are present in blood of asthmatics $(6,19)$. Importantly, PTGDR 2 mRNA and percentage of CRTh2 ${ }^{+}$cells in BAL have been reported to be highest in patients with severe asthma (20). Furthermore, asthma exacerbations and poor asthma control have been associated with higher CRTh2 levels. In addition, nucleotide polymorphisms in PTGDR2 have been linked with increased risk of asthma (21-23), and small-molecule inhibitors of CRTh2 signaling are currently under investigation as candidate therapeutics for asthma (24).

In light of these findings, we hypothesized that, as opposed to inhibiting PGD2-mediated CRTh2 activation, therapeutic depletion of CRTh2 $2^{+}$cells would eliminate many of the sources of pathogenic cytokines and mediators, including those targeted by either anti-IL13 or anti-IL5/IL5RA therapies, as well as those produced by Th2 and ILC2 cells that may underlie IL13/IL5-independent disease processes such as IL4, IL9, leukotrienes, and other mediators. Moreover, through the depletion of memory Th2 cells responsible for asthma chronicity (1-3), this therapeutic approach offers the potential for persistent efficacy. Here, we describe the identification, characterization, and humanization of mouse anti-hCRTh2 19A2 mAbs with effector functions that deplete eosinophils, basophils, ILC2 cells, and IL4-producing CD4 ${ }^{+} \mathrm{T}$ cells from tissues or circulation in multiple in vivo models. The humanized hCRTh2-specific antibody h19A2 may afford a broadly efficacious therapy to the treatment of human asthma and other allergic diseases.

\section{Results}

CRTh2 is expressed on human type-2 immune cells in lung and lymphoid organs. To further characterize hCRTh2, we profiled human peripheral blood leukocytes (PBLs), Th cell cultures, and tissues for their protein expression of hCRTh2. We confirmed by flow cytometry that hCRTh2 is highly expressed on PBL-derived eosinophils, basophils, and CD161 lin- ILC2s, as well as on in vitro-differentiated Th2 but not Th1 cells (Figure 1A). By comparison, CRTh2 was not detected on $\mathrm{CD}^{+}$or $\mathrm{CD} 8^{+} \mathrm{T}$ cell subsets including Tregs, monocytes, neutrophils, or B cells (Supplemental Figure 1A; supplemental material available online with this article; doi:10.1172/jci.insight.86689DS1). To verify the presence of CRTh2 ${ }^{+}$cells in human tissues, we raised 2 rabbit anti-CRTh2 mAbs against a peptide derived from the cytoplasmic tail of hCRTh2 (mAbs 81.12.4 and 108.1.6), both showing comparable immunoreactivity by IHC on human tissues (not shown). IHC on a panel of human tissues (Supplemental Table 1) revealed anti-hCRTh2-immunoreactive leukocytes scattered in normal lymph nodes (LNs) and spleens, as well as airways of asthmatics (Figure 1B). Several other tissues also contained hCRTh2+ immune cells, including skin dermal perivascular immune infiltrates, small intestinal lamina propria, and thymic cortex (Supplemental Figure 1B). Given the central role of tissue mast cells to asthma pathogenesis and difficulty evaluating them by flow cytometry, we assessed CRTh2 expression on tryptase-positive mast cells by dual label immunofluorescence microscopy in a variety of human tissues including airways. No convincing evidence of CRTh2 expression on tryptase-positive tissue mast cells was observed (Supplemental Table 2 and Supplemental Figure 1C), despite observing expression in human BM-derived mast cells in vitro (data not shown). Overall, these data confirm that hCRTh2 ${ }^{+}$is expressed only on select immune cells implicated in Th2 inflammatory responses and can be detected in human tissues at relevant sites. 


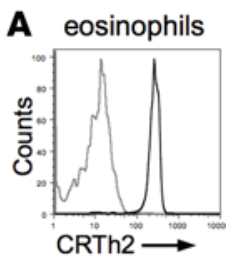

B
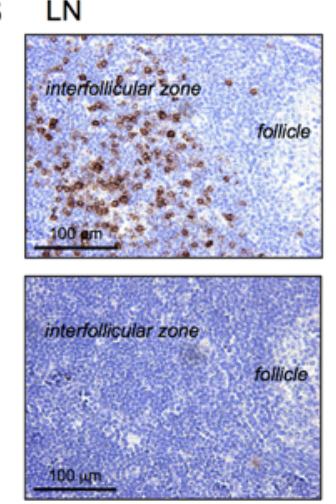

basophils

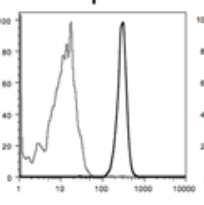

Spl
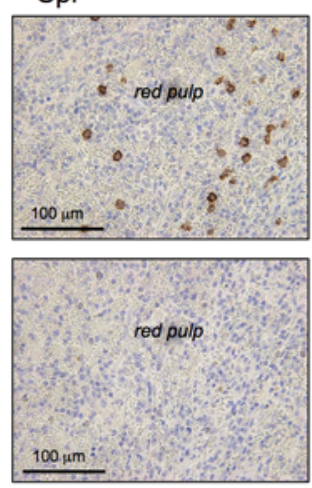

ILC2 cells

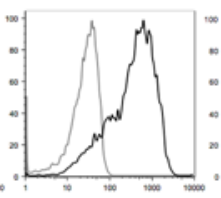

$\underbrace{}_{n=\infty}$

Th1

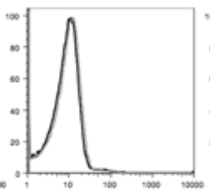

Airway
Th2

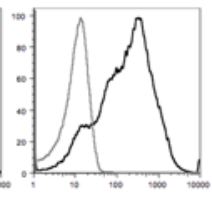

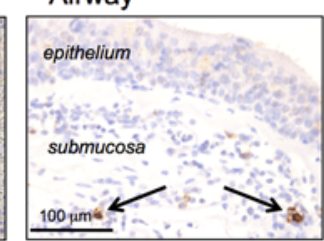

clone

81.12.4

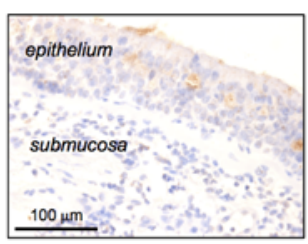

Isotype
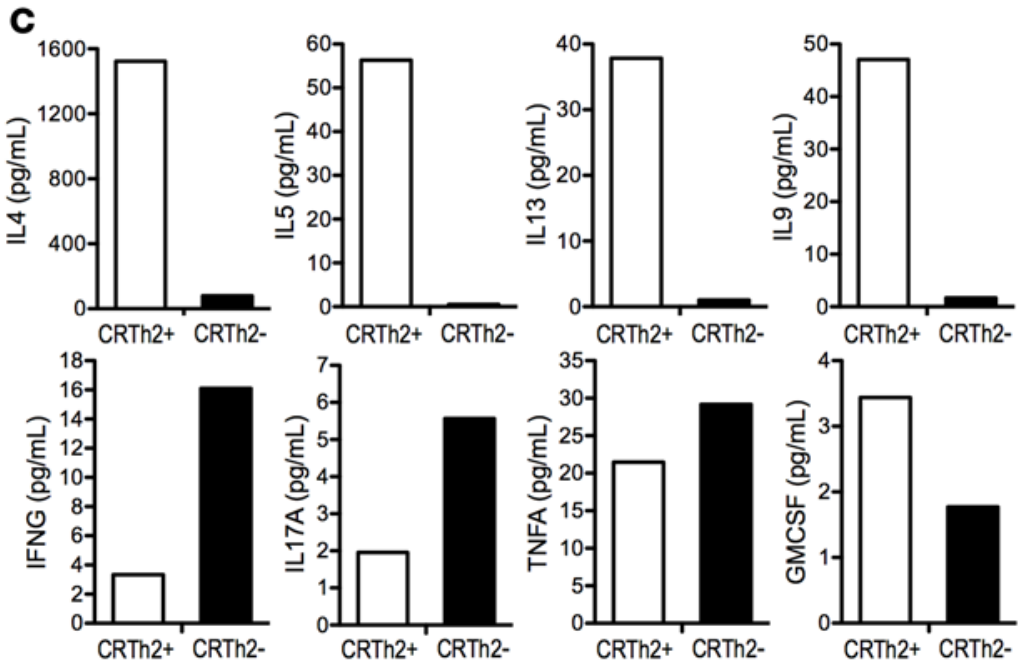

Figure 1. CRTh2 is expressed on human type- 2 immune cells and CRTh2 ${ }^{+}$memory $C D 4^{+} T$ cells are the major producers of CD4 ${ }^{+}$T cell-derived Th2 cytokines. (A) CRTh2 expression was assessed on human leukocyte populations and in vitro-polarized Th cells by flow cytometry with anti-CRTh2 Abs (clone BM16, black line) compared with isotype control Ab (gray line). (B) Expression of CRTh2 in human tissues was examined by IHC using rabbit anti-hCRTh2 mAb (clone 81.12.4) and, for comparison, an isotype control antibody. (C) Graphs show levels of Th2 (IL4, IL5, IL13, IL9) and Th1/Th17 (IFNG, IL17A, TNFA, GMCSF) cytokine production in supernatants of CRTh2+CD45RO ${ }^{+}$and CRTh2-CD45RO+ memory CD4 $T$ cells. T cell subsets were sorted by flow cytometry from human PBMCs, and equal numbers were stimulated with anti-CD3 and anti-CD28 antibodies for 48 hours at $37^{\circ} \mathrm{C}$. (A-C) Data shown are representative of at least 2 independent experiments. PBMC, peripheral blood mononuclear cell.

Memory $C D 4^{+} T$ cells are the major producers of $C D 4^{+} T$ cell-derived Th2 cytokines. CRTh2 was reported to be the most reliable marker to identify human Th2 $\mathrm{CD} 4^{+}$memory cells (13). To further characterize the relative production of the array of Th2 cytokines by hCRTh2+ versus hCRTh2- memory CD $4 \mathrm{~T}$ cells, we sorted hCRTh2 ${ }^{+}$and hCRTh2 ${ }^{-}$subsets of $\mathrm{CD}^{+}{ }^{+} \mathrm{CD} 45 \mathrm{RO}^{+} \mathrm{T}$ cells from the peripheral blood of atopic donors and stimulated them for 48 hours with anti-CD3/CD28 mAbs. Consistent with hCRTh2 being a marker for $\mathrm{Th} 2 \mathrm{CD}^{+} \mathrm{T}$ cells, the hCRTh2 ${ }^{+}$memory $\mathrm{CD} 4^{+} \mathrm{T}$ cell subset produced the vast majority of the classical Th2 cytokines IL4, IL5, IL13, and IL9 with negligible production by the hCRTh2- memory $\mathrm{CD}^{+} \mathrm{T}$ cell subset (Figure 1C). In contrast, hCRTh2- cells secreted large amounts of the Th1 cytokine IFNG. The hCRTh2 $2^{+}$subset also secreted GMCSF, TNFA, and notably IL17A, in agreement with previous reports that hCRTh $2^{+}$cells can also display features of $\operatorname{Th} 17$ cells $(25,26)$. These findings demonstrate that 

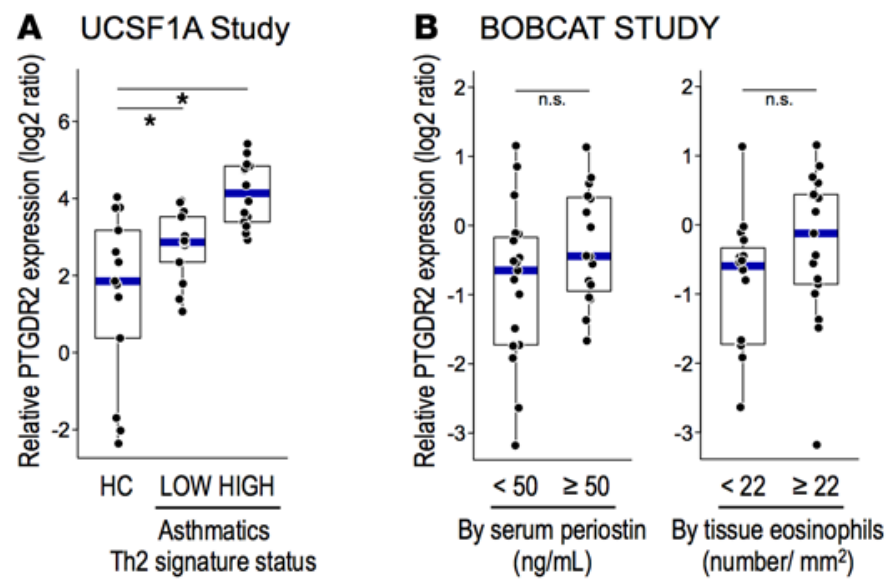

Figure 2. PTCDR2 gene expression is increased in Th2-high asthma. (A) Relative PTCDR2 expression in epithelial brushings of patients with mild/moderate asthma and healthy volunteers (HC). The Th2 signature status of asthmatics was determined as described (27). (B) Relative PTGDR2 expression in bronchial biopsies of patients with severe asthma from the Bronchoscopic Exploratory Research Study of Biomarkers in Corticosteroid-refractory Asthma (BOBCAT). Patients were grouped based on their serum periostin levels or their tissue eosinophil counts as described (28); healthy controls were not examined in the BOBCAT study. (A and B) Gene expression is expressed as the $\log _{2}$ ratio of test (samples) versus reference (universal human reference) channels. Gene expression data were plotted using boxplots overlaid with all observed data points. The box corresponds to the first quartile, median (blue), and third quartile. The whiskers extend to the most extreme data point that is no more than 1.5 times the interquartile range from the box; ${ }^{*} P \leq 0.05$ (2-tailed Student's $t$ test). PTCDR2, prostaglandin D2 receptor.

hCRTh2 ${ }^{+}$memory $\mathrm{CD} 4^{+} \mathrm{T}$ cells are the major producers of inflammatory Th2 cytokines IL4, IL5, IL9, and IL13 among memory CD4 $4^{+} \mathrm{T}$ cells.

CRTh2 (PTGDR2) gene expression is increased in Th2/eosinophilic-high asthma. To examine relative hCRTh2 transcript expression in subpopulations of asthma based on their eosinophilic or type-2 inflammatory state, we determined hCRTh2 expression in RNA prepared from bronchial biopsies of previously collected and categorized asthma patient samples $(9,27,28)$. In mild/moderate asthmatic patients, there was a statistically significant increase (6.4-fold) of gene expression of PTGDR2 encoding hCRTh2 in patients who were previously categorized as Th2-high based on a composite Th2 gene signature, consisting of POSTN, CLCA1, and SERPINB2, compared with healthy controls. Interestingly, there was also statistically significant increase in $P T G D R 2$ transcripts (2.5-fold) in patients with Th2-low phenotype compared with healthy controls, although not as pronounced (Figure 2A). Patients with severe asthma that showed evidence of eosinophilic airway inflammation relative to those with low tissue eosinophil counts — or similarly, patients who demonstrated higher than $50 \mathrm{ng} / \mathrm{ml}$ serum concentrations of the $\mathrm{Th} 2$ inflammatory marker periostin while not reaching statistical significance - also showed a trend of increased gene expression of hCRTh2 (1.5-fold) in bronchial biopsies (Figure 2B). These data indicate that PTGDR2 gene expression is elevated in subpopulations of asthma patients with a Th2/eosinophilic-high airway phenotype.

Generation and in vitro characterization of anti-hCRTh2 antibodies. We generated $\mathrm{mAbs}$ against the extracellular region of hCRTh2 by immunizing Balb/c mice with murine 300.19 pre-B cells engineered to express hCRTh2. We identified several hCRTh2-specific antibodies and selected clones 19A2 and 8B1 for subsequent studies. Both hCRTh2-specific mAbs bind in a dose-dependent manner to 293 or 300.19 cell transfectants that express surface hCRTh2 but not to hCRTh2-negative WT cells (Figure 3A and Supplemental Figure 2). In addition, recombinantly expressed $19 \mathrm{~A} 2$ and $8 \mathrm{~B} 1$ bind hCRTh2 on primary human blood basophils and eosinophils in a dose-dependent manner (Figure 3B). Single-arm (Fab fragment) or whole IgG hCRTh2specific antibodies bind to surface-expressed hCRTh2 on 293 cells with nanomolar affinity (Table 1).

To assess the function-blocking activity of the anti-hCRTh2 mAbs in response to PGD2 engagement, PGD2-induced calcium mobilization of in vitro polarized Th2 cells was measured in the presence of anti-hCRTh2 or control antibodies. Calcium flux triggered by PGD2 was completely prevented by preincubation of cells with $8 \mathrm{~B} 1 \mathrm{mAb}$, while treatment with $19 \mathrm{~A} 2 \mathrm{mAb}$ did not significantly affect $\mathrm{Ca}^{2+}$ flux (Figure 3C). These data indicate that 19A2 does not prevent PGD2-mediated responses, in contrast to 8B1, which can block PGD2-mediated activation of hCRTh2 $2^{+}$cells. 
A

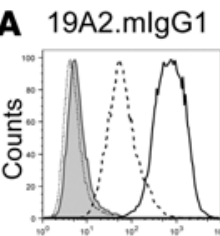

8B1.mlgG2b

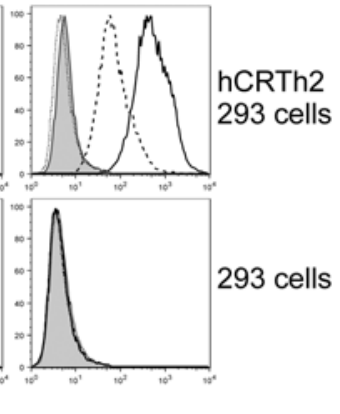

B $19 \mathrm{~A} 2 . \mathrm{mlgG} 2 \mathrm{a}$

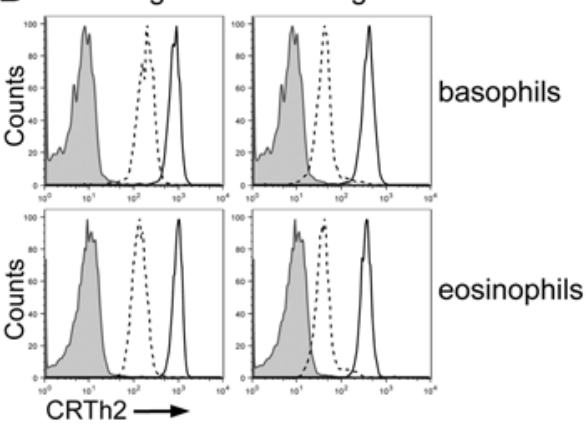

C

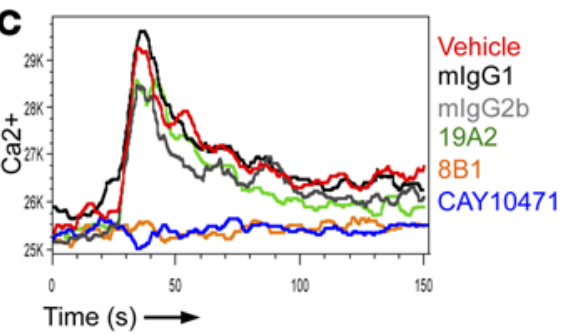

Figure 3. hCRTh2-reactive antibodies 19A2 and 8B1 specifically bind hCRTh2. (A) Flow cytometry analyses of mouse anti-hCRTh2 Abs $19 \mathrm{~A} 2$ and 8B1 binding to hCRTh2 expressed on 293 cells but not mock-transfected WT 293 cells. AntiCRTh2 Ab concentrations were $20 \mu \mathrm{g} / \mathrm{ml}$ (solid line), $2 \mu \mathrm{g} /$ $\mathrm{ml}$ (dashed line), and $0.2 \mu \mathrm{g} / \mathrm{ml}$ (dotted line); isotype control Ab was $20 \mu \mathrm{g} / \mathrm{ml}$ (tinted histogram). (B) Reactivity by flow cytometry of mouse anti-hCRTh2 antibodies 19A2 and 8B1 with primary human basophils or eosinophils from peripheral blood leukocytes. Anti-CRTh2 Ab concentrations were $10 \mu \mathrm{g} /$ $\mathrm{ml}$ (solid line) or $1 \mu \mathrm{g} / \mathrm{ml}$ (dashed line); isotype control Ab was $10 \mu \mathrm{g} / \mathrm{ml}$ (tinted histogram). (C) Anti-CRTh2 Ab 8B1, but not 19A2, prevents PGD2-induced calcium mobilization. Calcium flux in response to PCD2 stimulation of in vitro polarized human Th2 cell cultures gated on CCR4 ${ }^{+} \mathrm{CCR6}^{-} \mathrm{CXCR}^{-} \mathrm{CD}^{+}{ }^{+}$ T cells was monitored by flow cytometry in the presence of anti-CRTh2 or isotype control antibodies at $100 \mathrm{nM}$. The CRTh2 receptor antagonist CAY10471 was included as a positive control. Data shown are representative of at least 3 independent experiments. PGD2, prostaglandin D2.

Generation of an hCRTh2 transgenic mouse model. To characterize the in vivo depleting capacity of therapeutic anti-hCRTh2 mAbs and the functional consequences of eliminating CRTh2 $2^{+}$cells, we generated a murine model with an integrated BAC encoding hCRTh2 with the aim to recapitulate the expression pattern of hCRTh2 in murine cells under its own locus control region. In this respect, based on RNA expression analyses and functional studies, murine CRTh2 is more broadly expressed compared with hCRTh2 (29). Protein expression of hCRTh2 was readily detected on basophils and eosinophils by flow cytometry but, as expected, not on neutrophils and B cells of hCRTh2.BAC.Tg mice compared

with littermate nontransgenic mice (Figure 4A). In normal mice, Th2 cells and ILC2 cells are very rare. We therefore triggered type- 2 memory $\mathrm{CD}^{+} \mathrm{T}$ cell and ILC2 generation by injecting papain or recombinant murine IL25 (mIL25) into mice, respectively. Type- 2 memory CD4 ${ }^{+} \mathrm{CD} 44^{\mathrm{hi}} \mathrm{T}$ cells were induced in draining LNs as indicated by their cell surface expression of IL17Rb. However, hCRTh2 could not be detected on these memory $\mathrm{CD} 4{ }^{+} \mathrm{CD} 44^{\text {hi }} \mathrm{T}$ cells (Figure $4 \mathrm{~B}$ ). In contrast, hCRTh2 was readily detected on inflammatory ILC2 after mIL25 treatment in both mesenteric LNs and lung (Figure 4C). The lack of expression on Th2 cells could be due to differences in transcriptional activity of PTGDR2 between species, the presence of mouse PTGDR2, enhancer elements required for efficient PTGDR 2 mRNA transcription not found within the $\mathrm{BAC}$ or the integration site of the PTGDR2 BAC. The latter was less likely because none of the founder lines expressed hCRTh2 on memory $\mathrm{CD} 4{ }^{+} \mathrm{CD} 44^{\text {hi }} \mathrm{T}$ cells after papain injection (data not shown).

IHC analysis using 81.12.4 mAb of tissues derived from these hCRTh2.BAC.Tg mice but not WT littermates confirmed expression of hCRTh2 similar to human tissue expression on scattered leukocytes in the interfollicular zones of LNs, the red pulp of the spleen, and the airway submucosa (Figure 4D). We also noted hCRTh2 ${ }^{+}$eosinophil-rich clusters in spleens of hCRTh2.BAC.Tg mice, indicative of extramedullary eosinopoiesis and/or increased eosinophil recruitment to these sites in this model. These data show that the protein expression pattern on innate immune cells (basophils, eosinophils, and ILC2s), but not Th2 cells in hCRTh2. BAC.Tg mice, is similar to human, indicating that this model is useful to explore depletion of innate immune cells by anti-hCRTh2 mAbs.

Anti-CRTh2 mAbs deplete basophils and eosinophils in hCRTh2.Bac. Tg mice. We assessed the ability of the hCRTh2-specific mAb 19A2 to deplete eosinophils and basophils under homeostatic conditions. For this purpose, 19A2 was expressed with a mIgG2a constant region, which best recapitulates hIgG1-hFc $\gamma \mathrm{RI} /$ $\mathrm{hFc} \gamma \mathrm{RIII}$ interactions (30). We used an afucosylated version of mIgG2a for these studies to maximize antibody-dependent cell cytotoxicity (ADCC) in anticipation that a therapeutic humanized version of 
Table 1. Binding affinities of h19A2 and parent anti-hCRTh2 antibodies to hCRTh2+ 293 cells, primary human eosinophils, and primary human basophils.

\begin{tabular}{|c|c|c|c|c|c|}
\hline & \multicolumn{2}{|c|}{ hCRTh2+ 293 cells } & hCRTh2+ & Eosinophils & Basophils \\
\hline & $\begin{array}{c}\text { Fab affinity } \\
K_{\mathrm{D}}(\mathrm{nM})\end{array}$ & $\begin{array}{c}\text { IgG affinity } \\
K_{\mathrm{D}}(\mathrm{nM})\end{array}$ & \multicolumn{3}{|c|}{ FACS IgG binding (nM) } \\
\hline $19 \mathrm{~A} 2$ & 17 & 2 & nd & nd & nd \\
\hline $8 \mathrm{~B} 1$ & 16 & 2.6 & nd & nd & nd \\
\hline h19A2 & 14 & nd & 19 & $18 \pm 2$ & $17 \pm 1$ \\
\hline
\end{tabular}

Binding affinities were determined using Scatchard or flow cytometry analyses. FACS IgC binding \pm SEM $(n=5)$ of h19A2 is indicated; nd, not determined.

this $\mathrm{mAb}$ may benefit from this feature.

Administration of a single i.v. dose of 19A2 at 100 or $20 \mu \mathrm{g} /$ mouse resulted in complete depletion of blood basophils and eosinophils in hCRTh2.BAC.Tg mice on day 3 after treatment (Supplemental Figure 3). Complete eosinophil depletion was also observed 7 days after 19A2 treatment in blood - as well as in spleen or BM- with the higher dose of 19A2, while the lower dose showed partial depletion/recovery (Supplemental Figure 3B). In comparison, basophils were completely eliminated in spleen and significantly depleted in BM 7 days after a single 100- $\mu$ g dose of 19A2, while basophil numbers in $20 \mu \mathrm{g}$-treated mice mostly had recovered in blood, spleen, and BM. These data indicated that anti-hCRTh2 19A2 mAbs efficiently deplete basophils and eosinophils from blood and tissues in a dose-dependent manner under homeostatic conditions.

Treatment with anti-hCRTh2 19A2 mAbs depletes innate immune cells and prevents Th2 inflammatory responses during $N$. brasiliensis infection. To assess whether 19A2 treatment depletes innate immune cells, including ILC2, and prevents Th2 cytokine-mediated responses under Th2 inflammatory conditions, we infected mice with the gastrointestinal nematode Nippostrongylus brasiliensis (N. brasiliensis). Nine days after primary infection with these helminthes, eosinophil, basophil, and ILC2 cell numbers were increased in blood, lung, or mesenteric LNs (Figure 5, A-C). Treatment with 19A2 completely depleted eosinophils from blood (Figure 5A). Importantly, administration of anti-hCRTh2 mAbs depleted innate immune cells from tissues: eosinophils were eliminated in lung, and ILC2 (with the majority being natural ILC2 [nILC2]; data not shown) were completely depleted in both lung and mesenteric LNs, the two major tissues of their expansion (Figure 5, B and C). Lung basophil numbers showed a reduction by over $80 \%$ in this experiment (Figure 5B), while similar experiments demonstrated complete depletion (data not shown). Innate immune cell depletion was accompanied by a potent reduction in Th2 cytokines in the BAL, with IL4 and IL13 being reduced by about $90 \%$ and IL5 by $47 \%$. Furthermore, production of IL6, a cytokine generated by ILC2 and eosinophils $(31,32)$ that may play a role in amplifying inflammation, was also inhibited by 95\% (Figure 5D). We also assessed in total lung gene expression of a select set of chemokines that have been associated with pulmonary type-2 inflammatory cell recruitment (Ccl2/Mcp1, Ccl3/Mip1a, Cc18/ Mcp2, Cc111/Eotaxin1, Cc117/Tarc, Ccl24/Eotaxin2). Gene expression of all chemokines tested except Ccl24 was significantly upregulated by infection (Figure 5G). Treatment with anti-hCRTh2 mAbs significantly diminished expression of $\mathrm{Ccl} 17$ important for $\mathrm{CCR} 4^{+} \mathrm{T}$ cell recruitment into airways (Figure 5G). Transcripts of the $\mathrm{CCR} 1^{+} / \mathrm{CCR}^{+}$macrophage-attracting chemokine $\mathrm{Ccl} 3$ were also significantly inhibited, while $C c l 2$-encoding chemokines attractant to $\mathrm{CCR} 2^{+}$monocytes showed no significant change. No significant difference was observed for the CCR3 ligands Ccl11 and Ccl24 important for eosinophil chemotaxis. Interestingly, transcripts of $\mathrm{Ccl}$, encoding an agonist for CCR8 in mouse (33), were upregulated in response to 19A2 treatment (Figure 5G). According to the crucial role for innate immune cells in host defense to $N$. brasiliensis, depletion of ILC2, basophils, and eosinophils prevented worm expulsion 9 days after infection (Figure 5E) but did not significantly alter serum IgE levels (Figure 5F) in agreement with previously published data (34-36). Moreover, these data indicate that anti-CRTh2 treatment is efficient in depleting ILC2, basophils, and eosinophils under Th2 inflammatory conditions and that hCRTh2.BAC.Tg mice provide a valuable model to study innate immune cell function. 

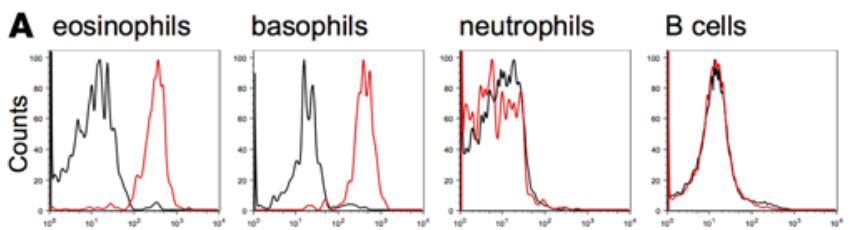

B Th2

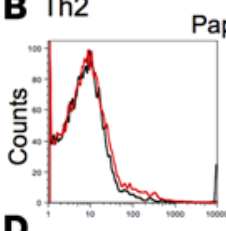

Papain

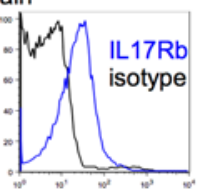

C ilLC2 cells

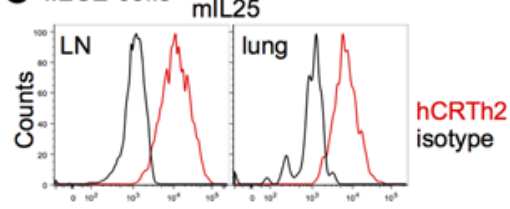

D LN

Spl
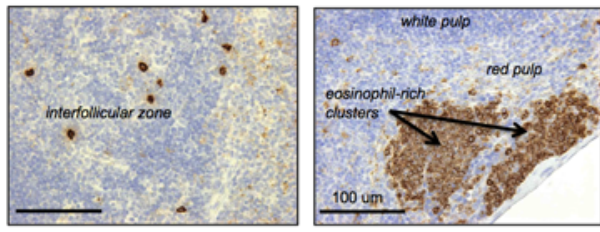

Airway

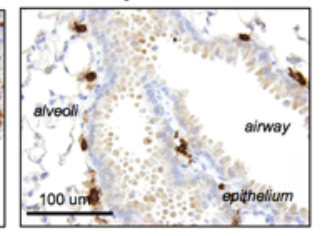

hCRTh2.Tg+
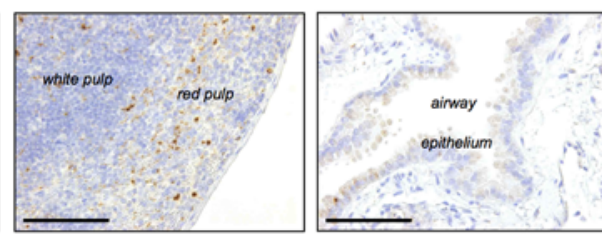

Figure 4. Human CRTh2.BAC.Tg mice express hCRTh2 on innate immune cells similarly to human cells. Flow cytometry (A-C) and IHC (D) analyses of hCRTh2 protein expression on immune cells under homeostatic ( $A$ and $\mathbf{D}$ ) and immunized (B and C) conditions. (A) Eosinophils (SSChiCCR3 ${ }^{+}$) and basophils (CD123+FceRI+) from blood and neutrophils (Gr1hiCD11b+) and B cells (B220+) from spleen were analyzed from hCRTh2.BAC.Tg mice (red line) or WT littermates (black line). (B and C) hCRTh2 expression (red line) compared with istotype control (black line) in (B) CD $4^{+} \mathrm{CD} 44^{\mathrm{hi}} \mathrm{T}$ cells (left histogram) from popliteal lymph nodes after treatment of hCRTh2.BAC.Tg mice with the Th2 polarizing agent papain for 2 weeks; as positive control for the induction of Th2 T cells, IL17Rb expression (blue line) compared with istotype control (black line) was monitored on the same subset of $\mathrm{CD4}^{+} \mathrm{T}$ cells (right histogram). (C) Inflammatory ILC2 (iILC2) from mesenteric LNs and lung after treatment of hCRTh2.BAC.Tg mice was monitored for 3 days with recombinant mIL25 i.p. (D) hCRTh2 in tissues from hCRTh2.BAC.Tg mice was examined using rabbit anti-hCRTh2 mAb (clone 81.12.4). Data shown are representative of at least 3 experiments.

Therapeutic treatment with anti-hCRTh2 $19 A 2 \mathrm{mAbs}$ depletes basophils and eosinophils from lung and inhibits IL4 production in murine allergic asthma. To determine the ability of anti-hCRTh2 mAbs to deplete in therapeutic regimens hCRTh2 ${ }^{+}$cells within lung tissue after onset of asthma disease, hCRTh2.BAC.Tg mice were sensitized with 2,4,6-trinitrophenyl-conjugated ovalbumin (TNP-OVA) in alum and aerosol challenged with TNP-OVA for 7 consecutive days commencing 5 weeks after the initial immunization. Because the model pathophysiology is highly Th2 cell dependent and Th2 cells do not express hCRTh2 in this model (Figure 1A), we focused on evaluating hCRTh2 ${ }^{+}$cell depletion rather than therapeutic efficacy, initiating therapeutic treatment with anti-hCRTh2 mAb after the 4th challenge, when lung inflammation is established. Flow cytometry revealed that anti-hCRTh2 $\mathrm{mAb}$ depleted basophils and eosinophils from lung tissue, while $\mathrm{CD}^{+} \mathrm{T}$ cell numbers were not affected (Figure 6A). Histological examination of eosinophils in lung tissue corroborated that eosinophils were absent from lung after 19A2 therapeutic dosing (Figure $6 \mathrm{~B})$. Furthermore, eosinophils were $100 \%$ depleted in the BAL fluid, whereas lymphocyte counts were not significantly changed (Figure 6C). Airway eosinophil and basophil depletion was accompanied in BAL by a complete reduction of IL4 and a partial decrease in IL13 with no changes in IL5, indicating that IL5 is predominantly produced by Th2 cells in this model (Figure 6D). Eosinophils were not only depleted in the pulmonary system but also in the spleen and blood, as observed under homeostatic conditions (Figure 6E; Supplemental Figures 3 and 4; data not shown).

To analyze the mechanisms important for in vivo depletion, we assessed the contributions of Fc receptor effector function through the use of an anti-hCRTh2 mAb with 2 mutations within the Fc effector domain (D265A and N297A, termed DANA). This mutant is well documented to abolish Fc $\gamma$ receptor (RI, RII, RIII, and RIV) binding but to retain normal in vivo pharmacokinetic properties because binding to FcRn is unaltered (30). Therapeutic treatment of hCRTh2.BAC.Tg mice with 19A2.DANA mAbs after induction of allergic asthma resulted in a significant loss of the ability of the mutant anti-hCRTh2 mAb compared with the WT 19A2 mAb to deplete lung eosinophils and basophils (Figure 6F), as well as BAL eosinophils and IL4 (Figure 6G). These data demonstrate that Fc receptor-mediated mechanisms play an important role in the elimination of eosinophils and basophils.

Therapeutic treatment with anti-hCRTh2 mAbs deplete Th2 cytokine-producing cells in SCID mice. Because hCRTh2.BAC.Tg mice lack induction of hCRTh2 on Th2 cells under the Th2 inflammatory conditions tested, we evaluated whether anti-hCRTh2 mAbs have the ability to deplete human peripheral blood mono- 

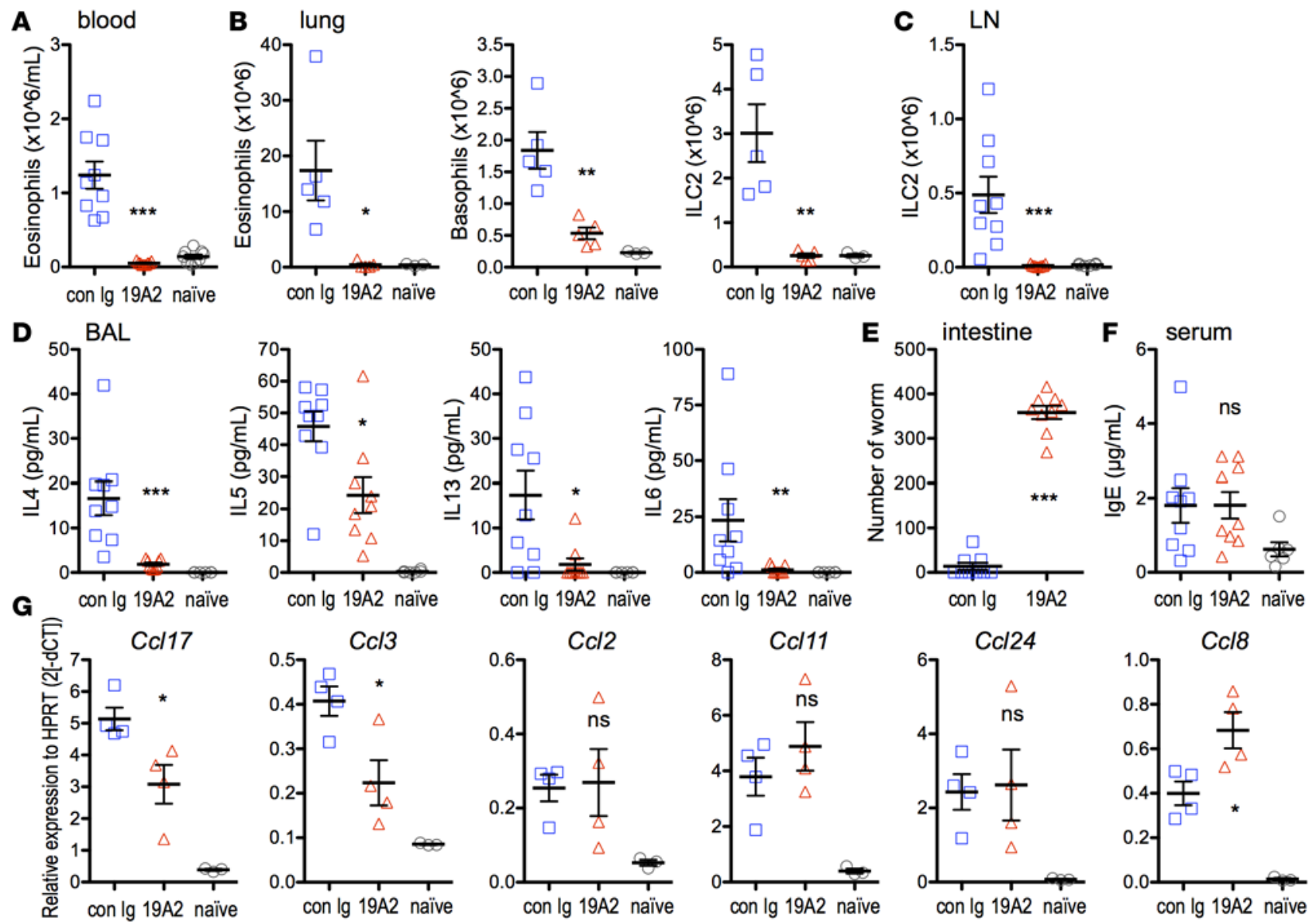

Figure 5. Treatment with anti-hCRTh2 Ab $19 A 2$ eliminates ILC2 cells, eosinophils, and basophils and reduces type-2 inflammatory responses during $\boldsymbol{N}$. brasiliensis infection. hCRTh2.BAC.Tg mice were infected with $N$. brasiliensis 9 days before analysis of tissue and blood samples. Mice were treated with $200 \mu \mathrm{g}$ of hCRTh2-specific afucosylated Ab 19A2 or control mlgG2a Ab on days -3, 0, 3, and 6. (A-C) Bar graphs show the absolute numbers of eosinophils, basophils, or ILC2 cells as determined by flow cytometry in blood (A), lung (B), or mesenteric LNs (C). (D) Th2 cytokine production was assessed by Luminex in BAL. (E) The dot plot shows the worm burden in the small intestine on day 9 after infection. (F) IgE was assessed by ELISA in serum. (G) Expression levels of chemokine genes were determined by quantitative PCR from RNA isolated from lung on day 9. Values are the relative expression of each gene to the control gene, HPRT. Results are mean \pm SEM; number of mice analyzed were for treatment groups $(n=9)$ and for naive mice $(n=6)$ except for lung were $n=5$ treatment, and $n=3$ naive mice were used for cell depletion analyses and the remaining mice ( $n=4$ and $n=3$, respectively) for gene expression studies. ${ }^{*} P \leq 0.05,{ }^{* *} P \leq 0.005$, ${ }^{* *} P \leq 0.0005$ (Dunnett's test). LN, lymph node; BAL, bronchoalveolar lavage; HPRT, hypoxanthine guanine phosphoribosyl transferase.

nuclear cell-derived (PBMC-derived) Th2 cells in a Th2-PBMC-SCID transfer model. For this purpose, naive $\mathrm{CD} 4 \mathrm{~T}$ cells isolated from $\mathrm{PBMCs}$ were cultured in vitro for 7 days under Th2 polarizing conditions that induce the expression of hCRTh2 (ref. 37 and Figure 1A) and adoptively transferred into SCID mice together with autologous PBMCs. Mice were treated with recombinant hIL4 and anti-hIFNG, plus antihIL12-blocking Abs, to further induce Th2 polarization in vivo as outlined in Figure 7A. Concomitant dosing of anti-hCRTh2 mAbs in comparison with isotype control mAbs significantly depleted IL4-producing $\mathrm{CD}^{+} \mathrm{T}$ cells, as assessed after ex vivo stimulation with PDBu plus Ionomycin of splenic cells on day 7 after transfer. In contrast, administration of 19A2 anti-hCRTh2 mAbs did not result in reduction of IFNG-producing $\mathrm{CD}^{+} \mathrm{T}$ cells (Figure 7B).

In summary, these data show that $19 \mathrm{~A} 2 \mathrm{mAbs}$ are potent in depleting hCRTh2 $2^{+}$basophils, eosinophils, ILC2, and Th2 T cells in vivo.

Generation and characterization of h19A2, a humanized hCRTh2-specific antibody. We developed a humanized hCRTh2-specific antibody that is suitable for human therapeutic administration in asthma and other allergic diseases by CDR grafting, followed by introduction of selected point mutations to enhance cross-reactivity with CRTh2 from primate species. The resulting humanized 19A2 hCRTh2-specific mAb, termed h19A2, expressed as a human IgG1 bound to hCRTh2 expressed on 293 cells but not WT 293 cells 

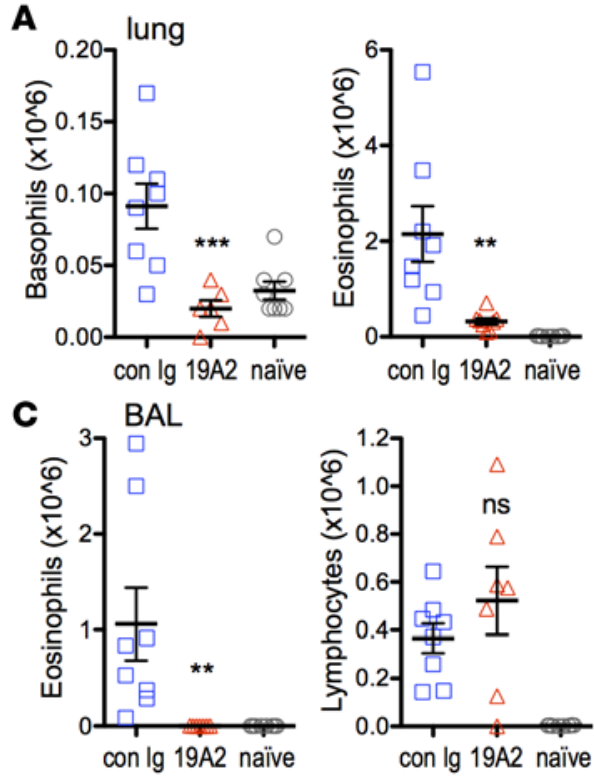

E

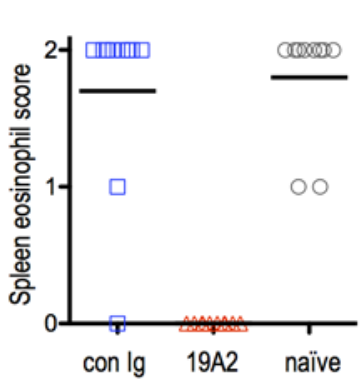

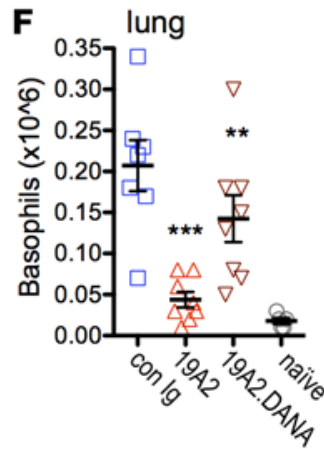

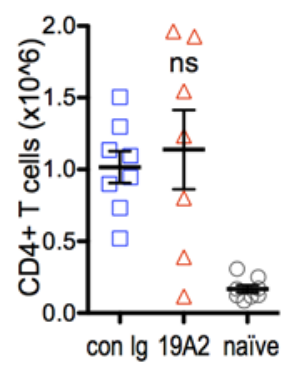

B

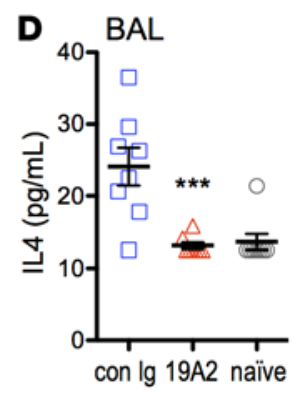

con Ig
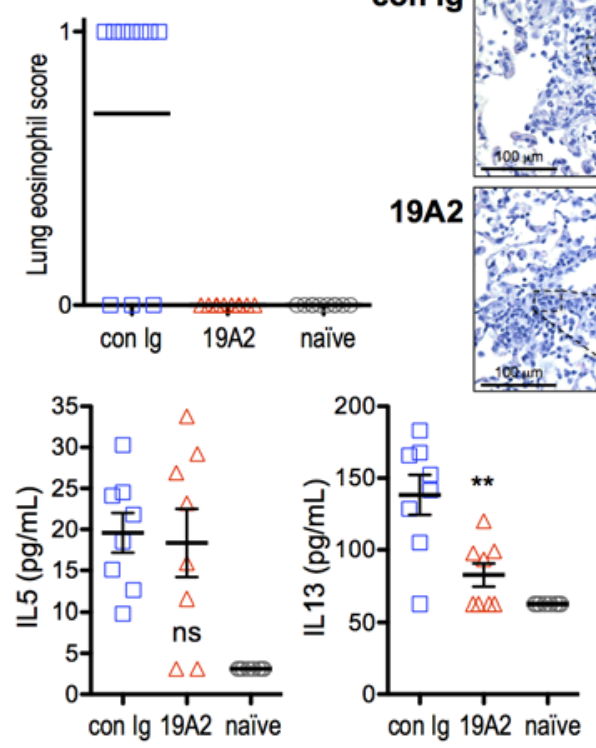

19A2

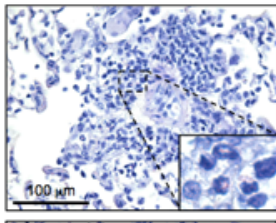

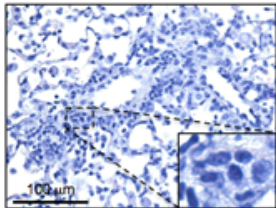
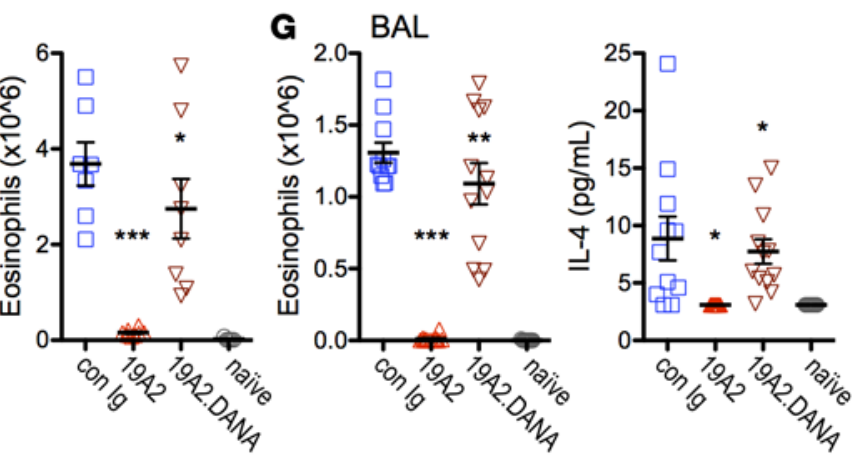

Figure 6. Therapeutic treatment with anti-hCRTh2 19A2 antibodies depletes basophils and eosinophils from lung and inhibits IL4 production in a mouse model of allergic asthma. hCRTh2.BAC.Tg mice were immunized with TNP-OVA/alum on day 0 and challenged with 7 daily aerosol administrations of TNP-OVA, starting on day 35 until day 41. (A-E) Mice were treated with $200 \mu \mathrm{g}$ of hCRTh2-specific afucosylated 19A2 or control mlgC2a Abs daily from day 38 through day 41. (A) Graphs show basophil, eosinophil, or CD4+ T cell numbers in lung as determined by flow cytometry. (B and E) Graphs show morphological scoring of eosinophil frequency in lung (B) and spleen (E); representative images of tissue sections are shown for lung (B). (C) Graphs show eosinophil or lymphocyte numbers in BAL as assessed by differential cell count. (D) The concentrations of IL4, IL5, and IL13 were determined by ELISA in BAL; the lower limit of detection was $6.25 \mathrm{pg} / \mathrm{ml}$ (IL4), $3.1 \mathrm{pg} / \mathrm{ml}$ (IL5), and $62.5 \mathrm{pg} / \mathrm{ml}$ (IL13). (F and G) Mice were treated with $200 \mu \mathrm{g}$ of hCRTh2-specific afucosylated 19A2, 19A2.DANA, or control mlgG2a Abs daily from day 38 through day 41. Treatment with afucosylated 19A2 Ab, but not with the 19A2.DANA mutant, depleted basophils and eosinophils from lung (F) and eosinophils from BAL (G, left panel) and prevented IL4 production in BAL (right panel); the lower limit of detection in the IL4 ELISA assay was $3.1 \mathrm{pg} / \mathrm{ml}$. Results are mean \pm SEM; number of mice per group were $n=7$ (control lg), $n=8$ (19A2, 19A2.DANA), or $n=5$ (naive). ${ }^{*} P \leq 0.05,{ }^{* *} P \leq 0.005,{ }^{* * *} P \leq 0.0005$ (Dunnett's test). TNP-OVA, $2,4,6$-trinitrophenylconjugated ovalbumin; BAL, bronchoalveolar lavage.

(Figure 8A) as well as primary human basophils and eosinophils (Figure 8B) in a dose-dependent manner. Scatchard and flow cytometry analyses showed respectively that the single-arm Fab fragment of 19A2 has nanomolar affinity for hCRTh2 (Figure $8 \mathrm{C}$ and Table 1) and that the whole IgG Ab exhibits nanomolar binding to hCRTh2 expressed on 293 cells, primary eosinophils, and primary basophils (Table 1). Humanized h19A2 mAb did not prevent PGD2-mediated reduction of forskolin-induced cAMP levels in hCRTh2 transfected 293 cells, while increasing concentration of a humanized version of 8B1 (h8B1) counteracted the PGD2-triggered decrease in cAMP (Figure 8D). These data demonstrate that h19A2, similar to its parent, is a nonfunction blocking antibody. Furthermore, h19A2 or h8B1 antibodies exhibited no agonistic activity on forskolin-induced cAMP levels in hCRTh2 transfected 293 cells, while PGD2 triggered a decrease in cellular cAMP levels (Figure 8E). Next, we compared the ADCC of the WT fucosylated h19A2 antibody with that of its afucosylated variant in an autologous ADCC assay using primary eosinophil and basophils as target cells and flow cytometry as a readout. The control for the ADCC assay was Rituximab that binds to human CD20. The afucosylated h19A2 mAb was approximately 10-fold more 

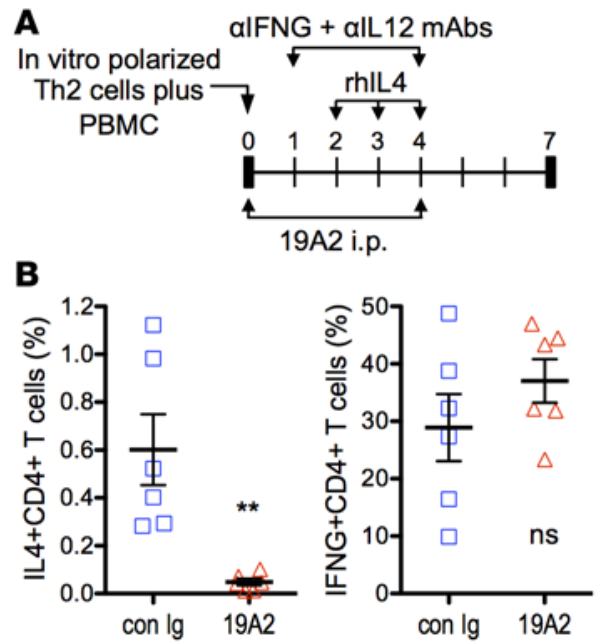

Figure 7. Therapeutic treatment with anti-hCRTh2 Ab $19 A 2$ specifically depletes IL4-producing Th2 $\mathrm{CD4}^{+} \mathrm{T}$ cells in a human Th2-PBMC-SCID mouse model. (A) Experimental design for the human Th2-PBMC-SCID mouse model. Sublethally irradiated SCID-beige mice ( $n=6$ per group) were injected with a mix of PBMC-derived in vitro-polarized human Th2 cells and autologous PBMCs. Mice were treated as outlined and described in Methods. (B) Graphs show the percentage of human IL4- or IFNG-producing human CD4+lin- T cells on day 7 as determined by flow cytometry and intracellular cytokine staining. Results are mean $\pm \mathrm{SEM}$. ${ }^{*} P \leq 0.005$ (Dunnett's test). PBMC, peripheral blood mononuclear cell.

potent against primary basophils and about 5.6-fold more potent against eosinophils than its fucosylated counterpart in the ADCC assay (Table 2), with comparable or slightly higher maximal ADCC activity as compared with the WT antibody (Figure 8F). Thus, afucosylation enhanced the in vitro ADCC potency of the hCRTh2-specific h19A2 mAb, consistent with its increased affinity for human Fc $\gamma R$ RIIIa receptors, which is typical for this change in glycosylation (ref. 38 and data not shown).

We assessed the ability of the humanized h19A2 mAb to deplete innate immune cells in hCRTh2.BAC. $\mathrm{Tg}$ mice under homeostatic conditions and therapeutically in the allergic asthma model. For the singledose homeostatic experiment, we used the native hIgG1 Fc; for the multi-dose experiments, we grafted the antibody with murine IgG2a Fc (reverse chimera) to avoid immunogenicity responses. Similar to what we previously observed with the murine parent antibody, administration of a single i.v. dose of h19A2 at 10 or $0.5 \mathrm{mg} / \mathrm{ml}$ resulted in complete depletion of eosinophils in blood, spleen, and BM 2 days after dosing (Supplemental Figure 5). The depletion was reversible and dose responsive, as eosinophils in the low-dose group started to recover in all 3 organs on day 7 and were fully recovered on day 14 . The high dose provided prolonged depletion with minimal recovery on day 7 in blood and on day 14 in blood and BM. Efficient depletion of eosinophils and basophils was also observed in allergic asthma in hCRTh2.BAC.Tg mice when treatment with h19A2 was started after onset of disease. Thus, therapeutic dosing of h19A2 completely depleted eosinophils from lung, BAL, and BM (Supplemental Figure 6). Similarly, h19A2 treatment was highly efficacious in depleting basophils from lung and BM.

Prolonged depletion of eosinophils with h19A2 mAbs does not significantly alter BM plasma cell numbers or serum Ig titers in hCRTh2.BAC.Tg mice. Production of IgE by plasma cells is a hallmark of allergic responses including asthma. Thus, it could be therapeutically desirable to reduce long-lived IgE-producing plasma cell numbers, while on the contrary, a broad reduction of long-lived plasma cells may constitute a safety concern by compromising general immunity. In this respect, eosinophils have been shown, at least partially, to control plasma cell persistence (31). To initially explore whether prolonged depletion of eosinophils by the clinical candidate h19A2 had the potential to affect plasma cell numbers under homeostatic conditions, we dosed hCRTh2.BAC.Tg mice weekly with h19A2 (reverse chimera) for 6 weeks at $15 \mathrm{mg} / \mathrm{kg}$ to maintain eosinophil depletion throughout the dosing period. Indeed, treatment with h19A2 but not with isotype antibodies nearly completely depleted eosinophils in the spleen and BM of mice 7 days after the final (sixth) dose (Figure 9, A and B). Intermittent analyses of blood samples from these mice at trough before the fourth antibody injections confirmed nearly complete depletion of eosinophils in blood (data not shown). Flow cytometry analyses and $\kappa$ light chain IHC revealed a mild trend but no significant depletion of plasma cells in BM and in spleen (Figure 9, A and B). Consistent with these negligible changes in plasma cell numbers, serum Ig concentrations of all isotypes tested were not altered by hCRTh2-specific depletion of eosinophils (Figure 9C). Therefore, our data indicate that treatment with anti-hCRTh2 in hCRTh2.BAC.Tg mice may only minimally reduce, but not eliminate, plasma cells in the BM. These findings are consistent with data reported by Cravedi et al. in eosinophil-deficient dblGATA1 B6 mice where plasma cell numbers showed a minimal to moderate reduction in BM and spleen and no differences in alloantibody titers after 
Table 2. Enhanced ADCC activity on primary human basophils and eosinophils of h19A2. af containing afucosylated hlgG1 compared with h19A2 containing WT hIgG1.

\begin{tabular}{|c|c|c|c|}
\hline & h19A2 (pM) & h19A2.af (pM) & Fold \\
\hline basophils & $4 \pm 2$ & $0.4 \pm 0.2$ & 10 \\
\hline eosinophils & $28 \pm 12$ & $5 \pm 1$ & 5.6 \\
\hline
\end{tabular}

immunization (39). Therefore, while reduction in eosinophils may moderately decrease BM plasma cells, it is unlikely to be clinically significant in lowering antibody titers (40).

\section{Discussion}

We have generated antibodies that deplete hCRTh2 ${ }^{+}$innate immune cells (eosinophils, basophils, and ILC2) and Th2 T cells, thereby reducing Th2 cytokines and chemokines that are essential for the propagation of Th2 inflammatory responses and, in particular, asthma. In vivo treatment with anti-CRTh2 mAbs efficiently and specifically eliminated hCRTh2 ${ }^{+}$cells from lung and lymphoid organs in multiple preclinical models through Fc receptor-mediated effector mechanisms. Targeting CRTh2+ Th2 cells and ILC2 for depletion in addition to eosinophils and basophils may specifically abolish the major sources of Th2 cytokines, chemokines, and other mediators driving human asthma and therefore offers the prospect of broader efficacy than antibodies that target a single cytokine pathway or pharmacologic blockade of CRTh2 activity with small molecule antagonists (41).

Phase $2 \mathrm{~b}$ clinical trials have shown that reduction of eosinophils with benralizumab, an afucosylated anti-IL5RA antibody that depletes blood and airway eosinophils, reduced asthma exacerbations in adults with uncontrolled eosinophilic asthma and baseline blood eosinophils of at least 300 cells per $\mu 1(8,42)$. In hCRTh2.BAC.Tg mice - which mirror hCRTh2 expression on eosinophils, basophils, and ILC2 treatment with anti-hCRTh2 19A2/h19A2 antibodies resulted in complete dose-dependent depletion of eosinophils from all tissues examined including lung, spleen, BM, and blood. Similarly, hCRTh2-specific antibodies eliminated, in a dose-dependent manner, basophils from all tissues except BM, where typically a small percentage of $\mathrm{Fc} \gamma \mathrm{RI}^{+} \mathrm{CD} 123^{+}$cell population remained; this suggests that immature basophils may not express hCRTh2 in these mice. DANA Fc modifications that inhibit 19A2 antibody binding to Fc $\gamma$ Rs largely prevented depletion of basophils and eosinophils in vivo, suggesting that ADCC mediates this cell elimination. Moreover, anti-CRTh2 h19A2 antibodies efficiently depleted primary human basophils and eosinophils in vitro from PBL in autologous ADCC assays; afucosylation further increased ADCC potency 5- to 10-fold. The in vitro ADCC potency of h19A2 appeared higher for human peripheral blood basophils than eosinophils (Table 2), despite that these cells express comparable hCRTh2 levels. A relatively higher efficiency for in vitro basophil depletion has also been reported for benralizumab (43). These findings together indicate that basophils are more sensitive to ADCC in vitro compared with eosinophils. Overall, our data show that anti-hCRTh2 treatment has the potential, similar to benralizumab, to efficiently eliminate pathogenic eosinophils and basophils in blood and in airways in asthma patients.

In contrast to benralizumab and other biological therapies, CRTh2-specific depleting antibodies directly target Th2 cells that are continually active in the airways, even when disease is quiescent (1-3). Accordingly, chronic activation of Th2 cells is sufficient for the induction of airway inflammation, chronic pathologic changes associated with asthma, and importantly, the persistence of asthma. Thus, even during periods of quiescent asthma disease, Th2 cytokines are produced in the airways, likely through the continued presence of airway memory Th2 T cells and prolonged antigen presentation by DCs $(1,44)$. In this respect, we showed that hCRTh2 is expressed on memory Th2 cells that are the major producers of classic T cell Th2 cytokines (IL4, IL5, IL9, IL13) as well as GM-CSF, which, in turn, may perpetuate local DC responses. Given this central role of memory Th2 cells to promote chronicity, it is conceivable that elimination of $\mathrm{CRTh}^{+}$memory Th2 cells may result in long-term disease-modifying effects. Unfortunately, we could not examine the full consequences of Th2 memory cell contributions in hCRTh2.BAC.Tg mice due to the lack of hCRTh2 expression on Th2 cells in these mice. Nevertheless, we could demonstrate that 
A hCRTh2
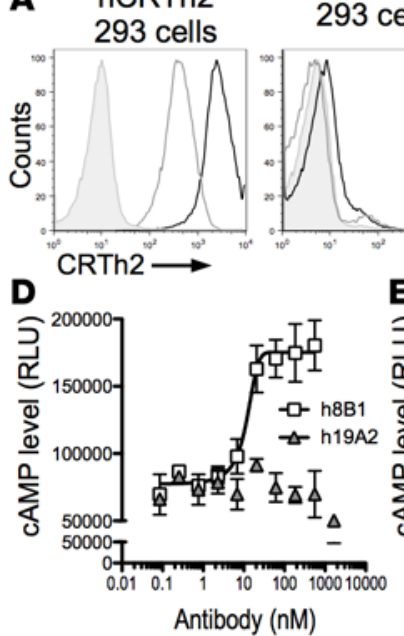

293 cells

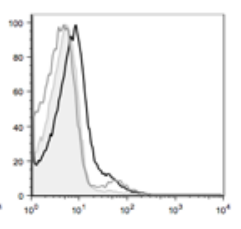

E
B

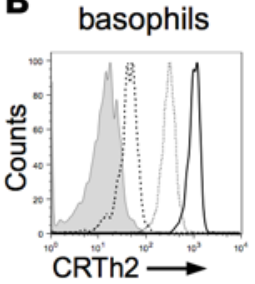

eosinophils

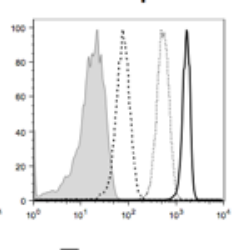

F basophils

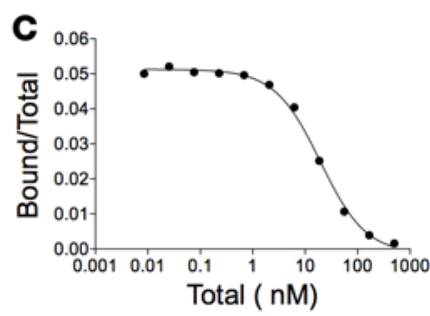

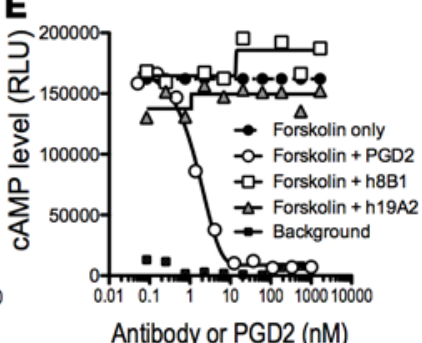

Antibody or PGD2 (nM)

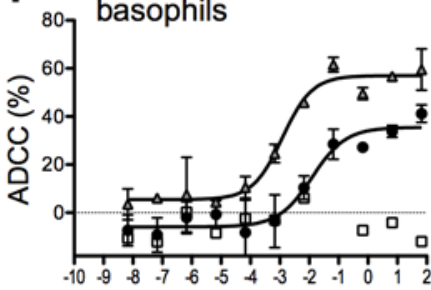

Antibody $(\log 10 \mathrm{nM})$

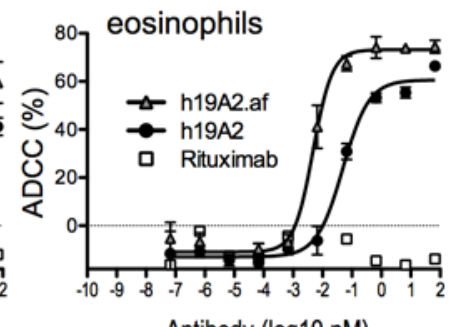

Figure 8. Characterization of h19A2, a humanized hCRTh2-specific Ab. (A) Flow cytometry analyses of humanized anti-hCRTh2 Ab h19A2 bound to hCRTh2 expressed on 293 cells but not mock-transfected WT 293 cells. Anti-CRTh2 Ab concentrations were $1 \mu \mathrm{g} / \mathrm{ml}$ (black line) and $0.1 \mu \mathrm{g} / \mathrm{ml}$ (gray line); isotype control Ab was $1 \mu \mathrm{g} / \mathrm{ml}$ (tinted histogram). (B) Reactivity by flow cytometry of humanized anti-hCRTh2 Ab h19A2 with primary human basophils or eosinophils from peripheral blood leukocytes. Anti-CRTh2 Ab concentrations were $5 \mu \mathrm{g} / \mathrm{ml}$ (solid line), $0.6 \mu \mathrm{g} / \mathrm{ml}$ (dotted line), and 0.06 $\mu \mathrm{g} / \mathrm{ml}$ (dashed line); isotype control Ab was $5 \mu \mathrm{g} / \mathrm{ml}$ (tinted histogram). (C) Scatchard analysis of the Fab fragment of humanized hCRTh2-specific Ab h19A2 binding to hCRTh2--transfected 293 cells. The mean binding affinity from 2 separate experiments is 11 nM. (D) Humanized h19A2 Ab does not block PGD2-mediated reduction of forskolin-induced cAMP levels in hCRTh2 ${ }^{+}$cAMP Hunter cells. The graph shows the measurement of intracellular CAMP levels in the presence of increasing concentrations of hCRTh2-specific antibodies h19A2 or h8B1 after cAMP levels were controlled with the concomitant administration of forskolin and PGD2. Results are mean of triplicates \pm SD. (E) Humanized h19A2 or h8B1 antibodies show no agonistic activity on forskolin-induced CAMP levels in hCRTh2 ${ }^{+}$cAMP Hunter cells. The graph shows the measurement of intracellular cAMP levels after induction of cAMP with forskolin in the presence of increasing concentrations of hCRTh2-specific antibodies h19A2 or h8B1, or with increasing concentrations of PGD2. (F) Afucosylation increases the potency of h19A2-mediated ADCC. Activity curves for hCRTh2-specific WT h19A2 (circles), afucosylated h19A2.af (triangles), and control Rituximab (squares) human IgG1 antibodies in an autologous flow cytometry-based ADCC assay using human primary basophils (left panel) and eosinophils (right panel) as target cells. A representative experiment is shown. The mean ADCC activity from 5 separate experiments is shown in Table 2. PGD2, prostaglandin D2; ADCC, antibody-dependent cell-mediated cytotoxicity.

human IL4-producing Th2 cells but not IFNG-producing Th1 cells are readily depleted by hCRTh2-specific antibody administration in a Th2-PBMC-SCID transfer model.

Depletion of CRTh2 ${ }^{+} \mathrm{T}$ cells may expand the pool of patients who benefit from a single therapy. Indeed, patients characterized by Th2-high asthma are not a homogeneous group, representing several different clinical and molecular phenotypes with divergent gene signatures $(10,11)$. CRTh2 $2^{+}$is expressed on subsets of IL17-producing Th2/Th17 cells and correlates with the presence of IL4/IL17 dual producing cells in asthmatics, while increased frequency of Th2/Th17 cells in BAL has been associated with poor lung function in these patients $(25,26)$. Additionally, our data indicate that CRTh2 is significantly expressed in patients with Th2-low asthma.

In addition to eliminating Th2 memory cells, anti-CRTh2 treatment may extend benefits to asthma patients through the depletion of ILC2 cells. While the contributions of ILC2 cells to human asthma are currently not well defined, ILC2s are increased in blood of asthma patients and contribute to type-2 lung inflammatory responses and airway hyper-responsiveness in mice infected with influenza virus and after allergen challenge $(19,45)$. During protective type- 2 immune responses after $N$. brasiliensis infection, ILC2 increase in lung and mesenteric LNs. We show that anti-CRTh2 treatment completely depleted ILC2 in tissues in N. brasiliensis-infected hCRTh2.BAC.Tg mice. Moreover, consistent with previously published data, hCRTh2-specific elimination of ILC2 together with basophils and eosinophils severely impaired the ability of CRTh2.BAC.Tg mice to expel $N$. brasiliensis $(34-36,45)$. These data demonstrate that anti-hCRTh2 treatment can block downstream mediators that effectively prevent type-2 functional responses.

Examination of effector pathways in response to hCRTh2-specific innate immune cell depletion in $N$. brasiliensis-infected hCRTh2.BAC.Tg mice demonstrated that concentrations of IL4 and IL13 were reduced around $90 \%$ in BAL on day 9 after helminth infection, while IL5 levels were only partially decreased and no changes in serum IgE were observed. These results are in agreement with previously published data that demonstrated that IL4/IL13 produced by innate immune cells, chiefly basophils and ILC2, are required for 

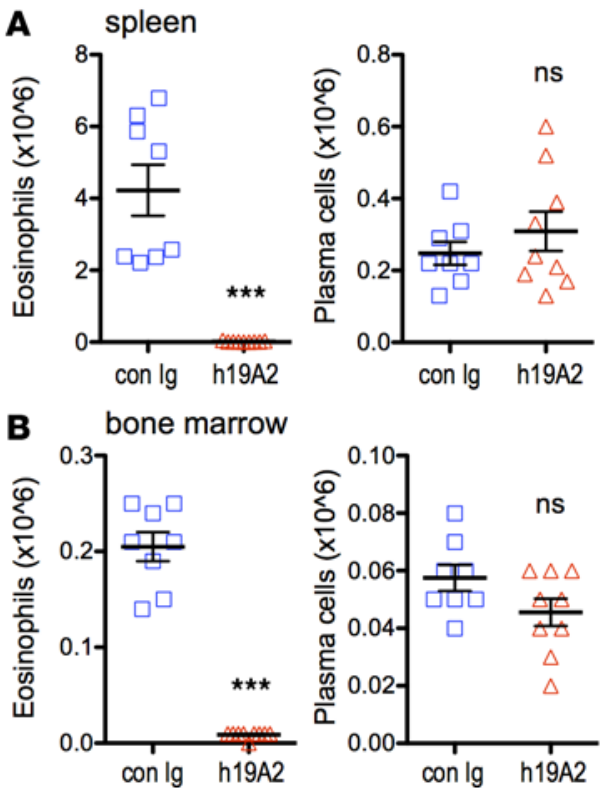
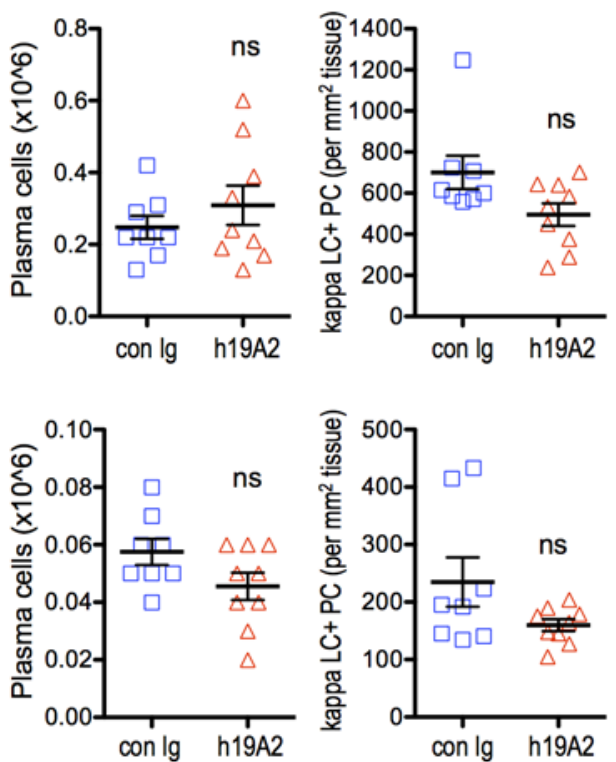
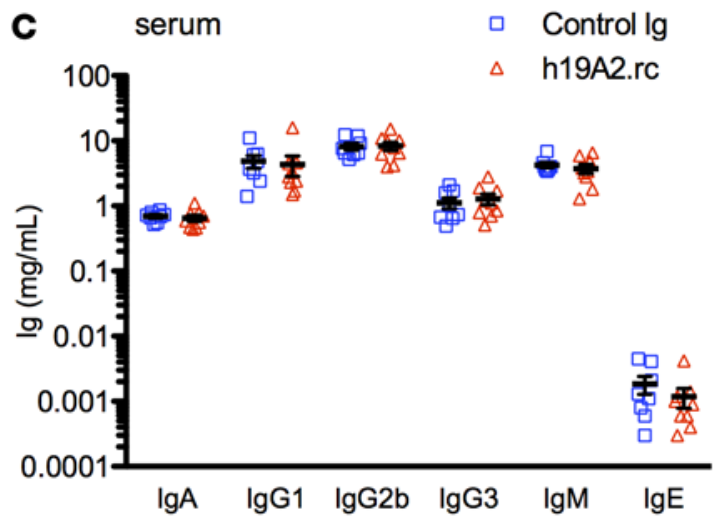

Figure 9. Prolonged depletion of eosinophils in hCRTh2.BAC.Tg mice does not significantly alter BM plasma cell numbers or serum Ig titers. hCRTh2.BAC.Tg mice were treated i.v. once per week for 6 weeks with $15 \mathrm{mg} / \mathrm{kg}$ of the murine reverse chimera ( $\mathrm{rc}$ ) of the humanized hCRTh2-specific h19A2 or control mlgC2a Ab. Cell numbers and Ig titers were determined on day 42, 7 days after the sixth dose of Ab. (A and B) Total number of eosinophils (left), B220+CD138+ plasma cells (middle), or $\kappa$ light chain-positive $\left(\mathrm{LC}^{+}\right)$plasma cells (right) in spleen (A) or BM (B) of h19A2.rc-treated (h19A2; $n=9$ ) or control mlgG2a-treated (con lg; $n=8$ ) unimmunized CRTh2.BAC.Tg mice. (C) Concentration of Ig in serum of treated mice. There was no statistical difference for the individual Ig isotypes between the h19A2.rc and control Ig treatment groups as determined by Dunnett's test. Isotype mlgG2a was not included in the comparison, as dosing antibodies contain this isotype. Results are mean \pm SEM. ${ }^{* *} P<0.0001$ (Dunnett's test); ns, not significant.

worm expulsion during primary infection and IL4/IL13 from T cells are not necessary for worm clearance but contribute to serum IgE (34-36). These data further indicate that IL5 production is the least dependent on innate immune cells in this model and is consistent with our findings in the asthma model where IL5 production was not impaired after therapeutic immune cell depletion. Thus, IL5 may provide a useful biomarker to monitor Th2 cell depletion.

In addition to classic Th2 cytokine, IL6 and several chemokine transcripts measured (Ccl17, Ccl3, Ccl2, Ccl11, and Ccl8) were also significantly increased after helminth infection. While IL6 protein, which can be produced by ILC2 and eosinophils $(31,32)$, was strongly reduced, $C c l 17$ and $C c l 3$ transcripts were partially inhibited. Nevertheless, given their respective role in $\mathrm{CCR} 4^{+} \mathrm{T}$ cell and CCR $1^{+} / \mathrm{CCR} 5^{+}$macrophage recruitment to the airways, a reduction in these chemokine transcripts may aid in disrupting pulmonary inflammation. Interestingly, Ccl8 mRNA was increased after innate immune cell depletion on day 9, when worms were still present in anti-hCRTh2 antibody-treated mice but not in control mice. As Cc18 has been implicated in signaling mucosal injury (46), it is possible that a compensatory mechanism in response to prolonged worm burden may cause this effect.

While the lack of hCRTh2 expression on Th2 T cells limits the exploration of the full reduction of hCRTh2 ${ }^{+}$cell-generated mediators in hCRTh2.BAC.Tg mice such as IL5 and IgE, these results demonstrate that anti-hCRTh2 treatment has the potential to significantly lower all major Th2 cytokines, as well as chemokines and other mediators. The combined effects of Th2 cytokine reduction may provide additional clinical benefits, given that functional redundancy is highly prevalent among Th2 cytokines (47).

In addition to Th2 cells, ILC2s, eosinophils, and basophils, mast cells have been implicated in asthma pathogenesis. A recent study has suggested that select human mast cells express CRTh2 protein, albeit the majority of CRTh2 protein may rest in intracellular pools (48). Using 2 independent antiCRTh2 mAbs qualified for CRTh2-specific detection in tissues, we have not been able to detect mast cell CRTh2 expression in a variety of mast cell-rich human tissues (Supplemental Table 2 and Supplemental Figure 1C). While we cannot exclude that rare human mast cells may express CRTh2 transiently under certain circumstances and thus may be sensitive to hCRTh2-specific depletion, we currently have no data to support this possibility. Nevertheless, some mast cells may be indirectly depleted through decreasing growth factors such as IL9. 
In future studies, anti-CRTh2 antibodies will need to be evaluated for their safety and pharmacodynamic and pharmacokinetic parameters in nonhuman primates. In this respect, it is noteworthy that, at present, eosinophil depletion and Th2 cytokine blockade has not been associated with major clinical safety issues preventing therapy in asthmatics $(6,49)$. hCRTh2-specific depletion of Th2 cells, eosinophils, basophils, and ILC2s may provide a valuable new therapeutic strategy to eliminate concomitantly several pathogenic cells and their mediators, and thus has the potential for superior and broader efficacy compared with agents that separately target either the eosinophilic component or single Th2 cytokines.

\section{Methods}

Supplemental Methods are available online with this article.

Generation of hCRTh2-expressing cell lines. Full-length hCRTh2 cDNA from Origene (Gene Bank NM_004778; encodes the more common alanine at position 204) was subcloned without or with N-terminal tags into the pRK5 vector. hCRTh2-expressing cells were generated by transfection of parental cells with hCRTh2-containing plasmids, using Fugene (Roche) for 293 cells (ATCC CRL-1573) and electroporation for 300.19 cells (Tufts University, Medford, Massachusetts, USA) as described (50). Surface expression of tagged or untagged hCRTh2 was confirmed by flow cytometry with anti-Flag mAb (clone M2, Sigma-A1drich), anti-gD Ab (clone 952, Genentech), or rat anti-hCRTh2 mAb (clone BM16, BD Pharmingen).

Generation of $h C R T h 2$-specific antibodies. Anti-CRTh2 antibodies for IHC were raised in rabbits against a synthetic peptide corresponding to the cytoplasmic tail of hCRTh2 (CAASPQTGPLNRALSSTSS). Hybridoma-derived $\mathrm{mAb}$ clones 81.12.4 and 108.1.6 were validated on $\mathrm{hCRTh} 2^{+}$and hCRTh2- cell pellets and tissues.

Anti-hCRTh2 depleting antibody hybridomas were generated from Balb/c mice (Charles River Laboratories) immunized with hCRTh2-expressing 300.19 cells. Antibodies were screened by flow cytometry using hCRTh2-expressing 293 or 300.19 cells and then primary human basophils and eosinophils. Light and heavy chains of clones 19A2 and 8B1 were subcloned into pRK5 vectors with heavy chains containing the WT or effector-deficient DANA mIgG2a Fc region. Anti-CRTh2 antibodies were produced in $\mathrm{CHO}$ cells using standard procedures. Murine 19A2 was humanized by grafting complementarity determining regions into the closest human consensus frameworks $\mathrm{K} 1 / \mathrm{VH} 3$ using gene synthesis (Genewiz). Back mutations were introduced to Vernier positions in order to keep the affinity to hCRTh2. Further engineering was carried out to keep the affinity to human but enable binding to other species. Afucosylated 19A2 antibodies were produced from a $\mathrm{FUT}^{-/-} \mathrm{CHO}$ cell line. Antibodies were purified by affinity chromatography using MabSelect SuRe (GE Healthcare) followed by size-exclusion chromatography using Superdex 200 (GE Healthcare). Protein identities were confirmed by mass spectrometry. Endotoxin levels were less than $0.5 \mathrm{EU} / \mathrm{mg}$.

Scatchard analysis. Iodinated anti-hCRTh2 was mixed with increasing concentrations of unlabeled anti-hCRTh2 antibodies and added to approximately $2.5 \times 10^{5}$ hCRTh2 transfected 293 cells in media containing $10 \mathrm{mM}$ HEPES, pH 7.4, 2\% FBS, and $40 \mu \mathrm{g} / \mathrm{ml} \mathrm{hIgG}$; incubated at room temperature for 2 hours; and filtered using a Multiscreen filtration plate (Millipore). Durapore membrane filters were counted using a Wallac Wizard 1470 Auto Gamma Counter, and binding affinity was determined using NewLigand software with standard Munson and Rodbard fitting algorithm (Genentech).

Human T helper cell polarization. Untouched naive $\mathrm{CD}^{+} \mathrm{T}$ cells were isolated from human PBMCs by MACS (Miltenyi Biotec) and cultured in DMEM media (10\% FBS, penicillin/streptomycin, $2 \mathrm{mM}$ L-glutamine, $50 \mu \mathrm{M}$ 2-ME, $1 \mathrm{mM}$ sodium pyruvate, and $1 \mathrm{mM}$ nonessential amino acids) with $10 \mu \mathrm{g} / \mathrm{ml}$ of platebound anti-CD3 $\mathrm{mAb}$ and $1 \mu \mathrm{g} / \mathrm{ml}$ soluble anti-CD28 mAb (BD Biosciences). Human Th polarization was stimulated as follows: Th1: $10 \mathrm{ng} / \mathrm{ml}$ recombinant human IL12 (rhIL12) plus $2 \mu \mathrm{g} / \mathrm{ml}$ anti-hIL4; Th2: 20 $\mathrm{ng} / \mathrm{ml} \mathrm{rhIL} 4$ plus $2 \mu \mathrm{g} / \mathrm{ml}$ anti-hIL12 and $5 \mu \mathrm{g} / \mathrm{ml}$ anti-hIFNG (cytokines from R\&D Systems, blocking antibodies from BD Biosciences). Two rounds of polarization were performed with the second restimulation in the presence of $1 \mu \mathrm{g} / \mathrm{ml}$ plate-bound anti-hCD3 $\mathrm{mAb}$ and $1 \mu \mathrm{g} / \mathrm{ml}$ soluble anti-hCD28 mAb.

CRTh2 $2^{+}$memory $C D 4^{+} T$ cell cytokine production. Untouched memory $\mathrm{CD}^{+} \mathrm{T}$ cells were isolated by MACS (Miltenyi Biotec) from human PBMCs of atopic donors with elevated serum IgE levels $(>200$ $\mathrm{kU} / 1$ ). Cells were stained at $37^{\circ} \mathrm{C}$ for 20 minutes with anti-CD45RO, anti-CD4 (both from BD Pharmingen), and anti-CRTh2 (clone BM16, Miltenyi Biotec) antibodies. CRTh2 ${ }^{+} \mathrm{CD} 45 \mathrm{RO}^{+} \mathrm{CD} 4^{+}$and $\mathrm{CRTh} 2{ }^{-} \mathrm{C}$ $\mathrm{D} 45 \mathrm{RO}^{+} \mathrm{CD}^{+}$memory $\mathrm{T}$ cells were sorted by FacsAria (BD Biosciences) to purities of above $98 \%$. Equal numbers of sorted cells were stimulated with $10 \mu \mathrm{g} / \mathrm{ml}$ of plate-bound anti-CD3 $\mathrm{mAb}$ and $1 \mu \mathrm{g} / \mathrm{ml}$ soluble 
anti-CD28 for 48 hours at $37^{\circ} \mathrm{C}$. Supernatants were collected and analyzed for cytokines using human BioPlex (Bio-Rad) antibody-immobilized beads and a Luminex 100 instrument.

Calcium mobilization assay. In vitro-polarized Th2 cells were incubated with $5 \mu \mathrm{M}$ indo-1/AM and $0.2 \%$ pluronic F127 (Molecular Probes) at $37^{\circ} \mathrm{C}$ for 30 minutes, washed, and stained with anti-CCR6, anti-CCR4, anti-CD4, and anti-CXCR3 mAbs (all from BD Biosciences) for 15 minutes. Washed cells were incubated with $1 \mu \mathrm{M}$ of anti-CRTh2 or isotype control antibodies at $37^{\circ} \mathrm{C}$ for 30 minutes and stimulated with 100 nM PGD2 (Sigma-Aldrich). Calcium release was monitored by flow cytometry.

Forskolin-induced cAMP assay. Recombinant cAMP Hunter CHO-K $\mathrm{C}_{1} \mathrm{CRTh} 2 \mathrm{Gi}$ cells (DiscoveRx) were cultured overnight at 10,000 cells/384-well. Culture medium was removed, and $10 \mu \mathrm{l} /$ well of testing antibody (serially diluted in PBS) was added and incubated for 30 minutes at $37^{\circ} \mathrm{C}$. Forskolin and PGD2 (Sigma-Aldrich) were added to $12.5 \mu \mathrm{M}$ and $4 \mathrm{nM}$, respectively. After 30 minutes at $37^{\circ} \mathrm{C}$, cAMP analysis was performed using a HitHunter cAMP XS+ kit (DiscoveRx) and an Envison chemiluminescence reader (Perkin Elmer).

Autologous ADCC assay. Human blood was obtained from healthy donors with Fc $\gamma$ RIIIA-158 V/F polymorphisms. After red blood cell lysis, $5 \times 10^{5}$ cells in RPMI with 10\% ultra-low IgG FBS (Hyclone) and $50 \mathrm{ng} / \mathrm{ml}$ of IL-2 (R\&D Systems) were incubated with serially diluted anti-CRTh2 or control antibodies at $37^{\circ} \mathrm{C}$ for 24 hours. Cells were washed and stained with anti-CD16, anti-CD123, anti-Fc $\gamma \mathrm{RI}$, and anti-CD19 followed by propidium iodide (BD Biosciences). Cell cytotoxicity was calculated as: percent of cytotoxicity $=100-([T / \eta] \times 100)$, where $T$ and $I$ are the counts of cells treated with test and isotype antibodies, respectively.

Generation of hCRTh2.BAC.Tg mice. A 171-kb human chromosome 11 fragment, containing the 1.2-kb CRTh2 gene flanked on either side by approximately $85 \pm 3 \mathrm{~kb}$ of genomic DNA, captured in a BAC-based plasmid (RPCI human BAC library 11, clone ID RP11-68H20, Invitrogen) was linearized and microinjected into blastocytes derived from C57BL/6 mice (Charles River Laboratories). The hCRTh2 transgene was detected by reverse transcription PCR (RT-PCR) from tail DNA. Seven founders were identified with similar hCRTh2 expression patterns on immune cells as tested by flow cytometry. One line was selected for use in all subsequent studies.

Immunizations. To determine hCRTh2 expression on Th2 cells, $50 \mu \mathrm{g}$ papain (Wako Pure Chemical Industries) in PBS was injected into one hCRTh2.BAC.Tg mouse hind footpad. Popliteal LN cells were collected 3 days later and analyzed by flow cytometry. To evaluate hCRTh2 expression on inflammatory ILC2, hCRTh2.BAC.Tg mice were treated i.p. with $0.5 \mu \mathrm{g}$ recombinant mIL-25 (Genentech) daily for 3 days. Lung and mesenteric LN cells were isolated and analyzed by flow cytometry.

$N$. brasiliensis infection. hCRTh2.BAC.Tg mice were infected on day 0 with 500 third-stage larvae (L3) of $N$. brasiliensis (R. Locksley, UCSF) isolated from the feces of infected Lewis rats (Charles River Laboratories) and treated i.p. with $10 \mathrm{mg} / \mathrm{kg}$ afucosylated 19A2 or control anti-ragweed antibodies (Genentech) every 3 days starting on day -3 and ending on day 6 . On day 9 , worm burden was assessed in the small intestine. Eosinophil numbers were evaluated in blood and ILC2 numbers in mesenteric LNs using flow cytometry. Lungs were used for either flow cytometry evaluation of cell composition or RNA isolation using MACS M tube homogenization (Miltenyi Biotec) and RNeasy Mini Kit (Qiagen) with on-column DNase digestion. Th2 cytokines in BAL fluid were measured by Luminex (Thermo Scientific) using Milliplex (EMD Millipore).

TNP-OVA asthma model. hCRTh2.BAC.Tg mice were sensitized i.p. on day 0 with $50 \mu \mathrm{g}$ TNP-OVA (Biosearch Technologies) in $2 \mathrm{mg}$ aluminum hydroxide in $100 \mu 1 \mathrm{PBS}$. Starting on day 35 after sensitization, mice were challenged daily for 7 days with aerosolized 1\% TNP-OVA in PBS for 30 minutes via a nebulizer. Mice were treated with anti-hCRTh2 or control antibodies in saline. On day 42, blood was collected via retro-orbital eye bleeds into heparin for flow cytometry evaluation and BAL for total and differential cell count and cytokine analyses. Mice were perfused with $20 \mathrm{ml}$ of PBS to clear the lungs of peripheral blood. Lungs were collected and processed without collagenase digest for flow cytometry. Cytokine concentrations in BAL were determined by ELISA (R\&D Systems).

Human Th2-polarized cell-SCID model. Human PBMCs were isolated by leukopheresis and Ficoll density gradient centrifugation (GE Healthcare) from atopic donors. Aliquots of PBMCs from the same donor were frozen for later transfer into SCID-beige mice (Charles River Laboratories). Untouched naive $\mathrm{CD} 4^{+} \mathrm{T}$ cells were isolated from PBMCs using the naive CD4 ${ }^{+} \mathrm{T}$ cell isolation kit II (Miltenyi Biotec) and stimulated for 3 days with $10 \mu \mathrm{g} / \mathrm{ml}$ plate-bound anti-CD3 and $1 \mu \mathrm{g} / \mathrm{ml}$ soluble anti-CD28 (BD Pharmingen) under Th2-polarizing 
conditions as described above. On day 7, $6 \times 10^{7}$ polarized T cells and $4 \times 10^{7}$ previously frozen PBMCs from the same donor were injected i.p. into SCID-beige mice that had been irradiated sublethally with 3.5 Gy from a cesium 137 source. Mice were treated with $110 \mathrm{mg} / 1$ polymyxin B and $1.1 \mathrm{~g} / 1$ neomycin in their drinking water throughout the study. To promote Th2 polarization, mice were treated with the following reagents i.p.: on day 0 and 3, $100 \mu \mathrm{g}$ anti-human IFNG plus $100 \mu \mathrm{g}$ anti-hIL12 antibodies; on days 1, 2, and 3, $100 \mathrm{ng}$ recombinant human IL4. Mice were dosed with $200 \mu \mathrm{g}$ afucosylated 19A2 or control antibodies on days 0 and 3. On day 7, spleens were harvested, and intracellular IL4 in human CD4 $4^{+} \mathrm{T}$ cells were determined by flow cytometry.

Flow cytometry. Nonspecific antibody binding was blocked with either $1 \mu \mathrm{g}$ of human IgG (Sigma-Aldrich) per $10^{5}$ cells for human or anti-CD16/CD32 (clone 2.4G2, BD Pharmingen) for mouse. Staining procedures were performed at room temperature. Dead cells were excluded with propidium iodide (Sigma-Aldrich), and cells were analyzed on a FACSCalibur or LSRII flow cytometer using CellQuest Pro (BD Biosciences) and FlowJo (Tree Star Inc).

Human blood was obtained from healthy donors, and rbcs were lysed with EL buffer (Qiagen). To evaluate hCRTh2 expression, human leukocytes were incubated with anti-CRTh2 (clone BM16 or 19A2) and the following BD Biosciences antibodies: anti-CD4, anti-CD8, anti-CD14, anti-CD16, anti-CD19, anti-HLADR, anti-CD123, and anti-Fc $\gamma$ RI. CRTh2 expression on human ILC2 was determined as previously described (14).

hCRTh2 expression on murine eosinophils, basophils, B cells, monocytes, and neutrophils from hCRTh2.BAC.Tg mice were determined with the following lineage antibodies: anti-CD123 and anti-F$c \gamma R I$ (eBioscience), anti-CCR3 (R\&D Systems), and anti-Gr1, anti-CD11b, and anti-B220 (all from BD Biosciences). mTh2-polarized cells were detected with anti-CD4 and anti-CD44; inflammatory ILC2 (KLRG1hiST2- $\mathrm{ICOS}^{+} \mathrm{Lin}^{-}$) and nILC2 (KLRG1intST2 $\left.{ }^{+} \mathrm{ICOS}^{+} \mathrm{Lin}^{-}\right)$were detected with anti-ST2 (MD Bioproducts), anti-KLRG1 (BD Horizon), anti-CD45 (BD Pharmingen), and anti-ICOS (eBiosciences) or alternatively anti-CD90.2 (BD Pharmingen) and antibodies against the following lineage markers: $\mathrm{CD} 3 \varepsilon$, CD4, CD8, TCR $\beta$, TCR $\gamma / \delta$, CD5, CD19, B220, CD11b, CD11c, TER119, Gr1, NK1.1, NKp46, DX5, Siglec-F (BD Pharmingen), F4/80 (Biolegend), and Fc $\gamma$ RI, CD123 (eBioscience); and plasma cells were detected with B220 plus CD138 (BD Horizon).

To detect intracellular cytokines in human $\mathrm{CD}^{+} \mathrm{T}$ cells from the PBMC-SCID experiment, splenocytes were stimulated ex vivo with $50 \mathrm{ng} / \mathrm{ml} \mathrm{PDBu}$ (Calbiochem) and $500 \mathrm{ng} / \mathrm{ml}$ ionomycin (Calbiochem) at $37^{\circ} \mathrm{C}$ for 4.5 hours; brefeldin $\mathrm{A}$ (BD Biosciences) was added for the final 3 hours. Cells were stained with anti-hCD4 and the following lineage markers: anti-mCD45, anti-mTer119, and anti-hCD19. Cells were fixed, permeabilized, and stained with anti-hIFNG and anti-hIL4 (BD Biosciences).

IHC and histology. IHC using rabbit anti-CRTh2 mAb (clone 81.12.4, Genetech) was performed on FFPE human or mouse tissue using Target Retrieval pH 9.0 Solution (DAKO) for antigen retrieval. Naive rabbit mAb DA1E (Cell Signaling Technology) was used in parallel as a nonspecific control. Detection employed goat anti-rabbit biotinylated secondary antibody (Vector Laboratories), followed by Streptavidin-HRP with tyramide signal amplification (PerkinElmer) and metal enhanced DAB visualization (ThermoFisher Scientific). IHC using rat biotinylated anti-mouse $\kappa$ light chain IgG (clone 187.1, BD Pharmingen) was performed on FFPE mouse tissue using Target Retrieval (DAKO), with naive rat biotinylated monoclonal IgG (clone R3-34, BD Pharmingen) in parallel as a nonspecific control. Detection employed Vectastain ABC-HRP (Vector Laboratories) and metal-enhanced DAB visualization (ThermoFisher Scientific). Whole-slide digital imaging was performed on an Olympus Nanozoomer at $\times 200$ magnification, and images were imported into MATLAB software (MathWorks) for morphometric quantitation of plasma cells per $\mathrm{mm}^{2}$ tissue using intensity and color thresholding. Automated recognition and enumeration of DAB-positive cells were further identified based on watershed transform, cell shape, and size criteria. Total tissue area was defined automatically using standard morphological operations. Image analysis segmentations were manually curated for accuracy.

Eosinophils in lung infiltrates were blindly scored on H\&E-stained sections as cells with characteristic pink-stained cytoplasmic granules and semisegmented ring-like nuclei using a Leica DM6000B microscope for readily identified (score $=1$ ) or rare/absent (score $=0$ ) eosinophils. Splenic red pulp eosinophils were scored on a 3-point scale (0: eosinophils rare/absent, 1: limited eosinophils in one or a few small red pulp nodules, and 2: many eosinophils in multiple larger nodules).

Serum immunoglobulin assays. Mouse immunoglobulins except IgE were analyzed on a Luminex FLEXMAP 3D System (Millipore), using a mouse Ig isotyping kit (MGAMMAG-300K; Millipore). Mouse IgE concentrations were measured by sandwich ELISA using rat anti-mouse IgE (clone R35-92; BD Biosciences) for capture and rat anti-mouse IgE-Biotin (clone R35-118; BD Biosciences) for detection. 
Quantitative PCR. Concentrations and integrity of RNA samples were determined using NanoDrop 8000 (Thermo Scientific). cDNA synthesis was performed on 100 ng total RNA using an iScript cDNA synthesis kit (Bio-Rad). Gene-specific preamplification was performed (Applied Biosystems) followed by RT-PCR using BioMark 96.96 Dynamic Arrays (Fluidigm Corporation). Data were collected using BioMark Data Collection Software, and Ct values were obtained using the BioMark RT-PCR Analysis Software (V.2.1.1, Fluidigm). Genes analyzed are summarized in Supplemental Table 3. The abundance $(\mathrm{dCt})$ relative to the house-keeping gene HPRT1 was calculated: $2^{-\mathrm{dCt}}$ with $\mathrm{dCt}=$ (average Ct gene - average Ct HPRT1). For statistical analyses, values below the lower limit of detection were set at $1 \mathrm{Ct}$ below the lowest recorded value.

Clinical samples. Bronchial biopsy RNA from mild-to-moderate nonsmoking asthma patients and healthy nonsmoking subjects was obtained from the UCSF Airway Tissue Bank, a specimen biorepository approved by the UCSF's Committee on Human Research, as reported previously (9, 27). Bronchial biopsy RNA and unstained histologic slides of human endobronchial biopsies were from severe, uncontrolled asthma patients from the Bronchoscopic Exploratory Research Study of Biomarkers in Corticosteroid-refractory Asthma (BOBCAT) (28). Gene expression microarrays were conducted and processed as previously described (27, 51). The microarray data are available in the Gene Expression Omnibus (GEO) repository (www.ncbi.nlm. nih.gov/projects/geo) under the accession number GSE23611 (Figure 2A) and GSE79145 (Figure 2B).

Statistics. Results were expressed as the mean \pm SEM unless specified. Dunnett's test was used to compare group means where all test groups were tested against a reference group. Groups from microarray analyses were compared using 2-tailed Student's $t$ test. Raw $P$ values are expressed for microarray data with no adjustments for multiplicity. $P$ values of less than 0.05 were considered to be significant.

Study Approval. Informed consent was obtained from all human subjects after the nature and possible consequences of the study were explained. Human tissues were acquired from commercial sources under warranty that appropriate IRB approval and informed consent was obtained. All mouse experiments were conducted in accordance with the NIH guidelines for the care and use of mice in research and approved by the Genentech Institutional Animal Care and Use Committee. Mice were bred under pathogen-free conditions.

\section{Author contributions}

TH, MH, Y. Shang, MZ, XW, DY, ZL, MS, EL, HN, Y. Shi, AK, DFC, NR, and JW designed and performed the experiments. All authors analyzed their respective data. ERC, MLM, and JP generated reagents and provided technical advice. MB, MX, and WPL established the Th2 animal models. JRA provided the clinical data and advice. JAH and IH designed the antibody development. JW designed cAMP, cytokine, and Ig assays. CDA designed the pathology studies. TH, Y. Shang, EL, DFC, MLM, JW, CDA, IH, and KR wrote the methods. TH, JRA, IH, and CDA edited the manuscript. KR designed and coordinated the research studies and wrote the manuscript.

\section{Acknowledgments}

We thank the following individuals for their help: Søren Warming, Michelle Bauer, and Merone RooseGirma (generation of CRTh2.Bac.Tg mice); Hongkang Xi, Neko Ota, Vladimir Ramirez-Carrozzi, Justin Lesch, and Jason DeVoss (ILC2 studies); Laurie Gilmour and Jim Cupp (flow cytometry); Rashi Takkar (cytokine analyses); Jianhua Tao, Felix Chu, and Charles Jones III (IHC); Shan Chung and Yugang Wang (ADCC assays); Haiqing Wang, Arthur Reyes, and Eric Stefanich (PK analyses); X.L. Xu (GRIP assays); Juan Zhang, Eric Suto, Nicole Valle, Victor Nunez, and Marjie Van Hoy (tissue harvest); Wenjun Ouyang, Lawren $\mathrm{Wu}$, and Flavius Martin (advice).

Address correspondence to: Karin Reif, KARBio, LLC, Phone: 650.291.7052; E-mail: KARBioSF@gmail. com. Or to: Cary Austin, Genentech Inc., 1 DNA Way, South San Francisco, California 94080, USA.; Phone: 650.225.7357; E-mail: austin.cary@gene.com.

Mercedesz Balazs' present address is: Amgen Inc., South San Francisco, California, USA.

Jo-Anne Hongo's present address is: JS Hongo Consulting Inc., Redwood City, California, USA.

Karin Reif's present address is: KARBio LLC., San Francisco, California, USA. 
1. Cohn L, Elias JA, Chupp GL. Asthma: mechanisms of disease persistence and progression. Annu Rev Immunol. 2004;22:789815.

2. Epstein MM. Targeting memory Th2 cells for the treatment of allergic asthma. Pharmacol Ther. 2006;109(1-2):107-136.

3. Robinson DS, et al. Predominant TH2-like bronchoalveolar T-lymphocyte population in atopic asthma. $N$ Engl J Med. 1992;326(5):298-304.

4. Bhakta NR, Woodruff PG. Human asthma phenotypes: from the clinic, to cytokines, and back again. Immunol Rev. 2011;242(1):220-232.

5. Sonnenberg GF, Artis D. Innate lymphoid cells in the initiation, regulation and resolution of inflammation. Nat Med. 2015;21(7):698-708.

6. Fajt ML, Wenzel SE. Asthma phenotypes and the use of biologic medications in asthma and allergic disease: the next steps toward personalized care. J Allergy Clin Immunol. 2015;135(2):299-310; quiz 1.

7. Simpson JL, Scott R, Boyle MJ, Gibson PG. Inflammatory subtypes in asthma: assessment and identification using induced sputum. Respirology. 2006;11(1):54-61.

8. Castro M, et al. Benralizumab, an anti-interleukin 5 receptor alpha monoclonal antibody, versus placebo for uncontrolled eosinophilic asthma: a phase 2b randomised dose-ranging study. Lancet Respir Med. 2014;2(11):879-890.

9. Woodruff PG, et al. T-helper type 2-driven inflammation defines major subphenotypes of asthma. Am J Respir Crit Care Med. 2009;180(5):388-395.

10. Modena BD, et al. Gene expression in relation to exhaled nitric oxide identifies novel asthma phenotypes with unique biomolecular pathways. Am J Respir Crit Care Med. 2014;190(12):1363-1372.

11. Wu W, et al. Unsupervised phenotyping of Severe Asthma Research Program participants using expanded lung data. J Allergy Clin Immunol. 2014;133(5):1280-1288.

12. Caruso M, Crisafulli E, Lizzio R, Polosa R. Biologic therapy for atopic asthma and beyond. Curr Opin Allergy Clin Immunol. 2013;13(6):677-685.

13. Cosmi L, Annunziato F, Galli MIG, Maggi RME, Nagata K, Romagnani S. CRTH2 is the most reliable marker for the detection of circulating human type $2 \mathrm{Th}$ and type 2 T cytotoxic cells in health and disease. Eur J Immunol. 2000;30(10):2972-2979.

14. Mjosberg JM, et al. Human IL-25- and IL-33-responsive type 2 innate lymphoid cells are defined by expression of CRTH2 and CD161. Nat Immunol. 2011;12(11):1055-1062.

15. Nagata K, et al. CRTH2, an orphan receptor of T-helper-2-cells, is expressed on basophils and eosinophils and responds to mast cell-derived factor(s). FEBS Lett. 1999;459(2):195-199.

16. Nagata K, et al. Selective expression of a novel surface molecule by human Th2 cells in vivo. J Immunol. 1999;162(3):12781286.

17. Mutalithas K, Guillen C, Day C, Brightling CE, Pavord ID, Wardlaw AJ. CRTH2 expression on T cells in asthma. Clin Exp Immunol. 2010;161(1):34-40.

18. Siracusa MC, Kim BS, Spergel JM, Artis D. Basophils and allergic inflammation. J Allergy Clin Immunol. 2013;132(4):789-801; quiz 788.

19. Bartemes KR, Kephart GM, Fox SJ, Kita H. Enhanced innate type 2 immune response in peripheral blood from patients with asthma. J Allergy Clin Immunol. 2014;134(3):671-678 e4.

20. Fajt ML, et al. Prostaglandin D(2) pathway upregulation: relation to asthma severity, control, and TH2 inflammation. J Allergy Clin Immunol. 2013;131(6):1504-1512.

21. Campos Alberto E, et al. The single nucleotide polymorphism CRTh2 rs533116 is associated with allergic asthma and increased expression of CRTh2. Allergy. 2012;67(11):1357-1364.

22. Huang JL, et al. Sequence variants of the gene encoding chemoattractant receptor expressed on Th2 cells (CRTH2) are associated with asthma and differentially influence mRNA stability. Hum Mol Genet. 2004;13(21):2691-2697.

23. Wang J, Xu Y, Zhao H, Sui H, Liang H, Jiang X. Genetic variations in chemoattractant receptor expressed on Th2 cells (CRTH2) is associated with asthma susceptibility in Chinese children. Mol Biol Rep. 2009;36(6):1549-1553.

24. Townley RG, Agrawal S. CRTH2 antagonists in the treatment of allergic responses involving TH2 cells, basophils, and eosinophils. Ann Allergy Asthma Immunol. 2012;109(6):365-374.

25. Irvin C, et al. Increased frequency of dual-positive TH2/TH17 cells in bronchoalveolar lavage fluid characterizes a population of patients with severe asthma. J Allergy Clin Immunol. 2014;134(5):1175-1186 e7.

26. Wang $\mathrm{YH}$, et al. A novel subset of CD4(+) T(H)2 memory/effector cells that produce inflammatory IL-17 cytokine and promote the exacerbation of chronic allergic asthma. J Exp Med. 2010;207(11):2479-2491.

27. Choy DF, et al. Gene expression patterns of Th2 inflammation and intercellular communication in asthmatic airways. $J$ Immunol. 2011;186(3):1861-1869.

28. Jia G, et al. Periostin is a systemic biomarker of eosinophilic airway inflammation in asthmatic patients. J Allergy Clin Immunol. 2012;130(3):647-654 e10.

29. Takeshita $\mathrm{K}$, et al. CRTH2 is a prominent effector in contact hypersensitivity-induced neutrophil inflammation. Int Immunol. 2004;16(7):947-959.

30. Chan AC, Carter PJ. Therapeutic antibodies for autoimmunity and inflammation. Nat Rev Immunol. 2010;10(5):301-316.

31. Chu VT, et al. Eosinophils are required for the maintenance of plasma cells in the bone marrow. Nat Immunol. 2011;12(2):151159.

32. Moro K, et al. Innate production of $\mathrm{T}(\mathrm{H}) 2$ cytokines by adipose tissue-associated c-Kit(+)Sca-1(+) lymphoid cells. Nature. 2010;463(7280):540-544.

33. Islam SA, et al. Mouse CCL8, a CCR8 agonist, promotes atopic dermatitis by recruiting IL-5 ${ }^{+} \mathrm{T}(\mathrm{H}) 2$ cells. Nat Immunol. 2011;12(2):167-177.

34. Huang Y, et al. IL-25-responsive, lineage-negative KLRG1(hi) cells are multipotential 'inflammatory' type 2 innate lymphoid cells. Nat Immunol. 2015;16(2):161-169.

35. Ohnmacht C, Schwartz C, Panzer M, Schiedewitz I, Naumann R, Voehringer D. Basophils orchestrate chronic allergic dermatitis and protective immunity against helminths. Immunity. 2010;33(3):364-374. 
36. Voehringer D, Reese TA, Huang X, Shinkai K, Locksley RM. Type 2 immunity is controlled by IL-4/IL-13 expression in hematopoietic non-eosinophil cells of the innate immune system. J Exp Med. 2006;203(6):1435-1446.

37. Langenkamp A, Nagata K, Murphy K, Wu L, Lanzavecchia A, Sallusto F. Kinetics and expression patterns of chemokine receptors in human CD4+ T lymphocytes primed by myeloid or plasmacytoid dendritic cells. Eur J Immunol. 2003;33(2):474482.

38. Junttila TT, et al. Superior in vivo efficacy of afucosylated trastuzumab in the treatment of HER2-amplified breast cancer. Cancer Res. 2010;70(11):4481-4489.

39. Cravedi P, Lessman DA, Heeger PS. Eosinophils are not required for the induction and maintenance of an alloantibody response. Am J Transplant. 2013;13(10):2696-2702.

40. Redfield RR, Abt PL, Naji A. A case for APRIL/BLyS directed therapy not eosinophil directed therapy for alloantibody elimination. Am J Transplant. 2014;14(2):492-493.

41. O’Byrne PM, Naji N, Gauvreau GM. Severe asthma: future treatments. Clin Exp Allergy. 2012;42(5):706-711.

42. Laviolette M, et al. Effects of benralizumab on airway eosinophils in asthmatic patients with sputum eosinophilia. $J$ Allergy Clin Immunol. 2013;132(5):1086-1096 e5

43. Kolbeck R, et al. MEDI-563, a humanized anti-IL-5 receptor alpha mAb with enhanced antibody-dependent cell-mediated cytotoxicity function. J Allergy Clin Immunol. 2010;125(6):1344-1353 e2.

44. Julia V, Hessel EM, Malherbe L, Glaichenhaus N, O'Garra A, Coffman RL. A restricted subset of dendritic cells captures airborne antigens and remains able to activate specific T cells long after antigen exposure. Immunity. 2002;16(2):271-283

45. Lund S, Walford HH, Doherty TA. Type 2 innate lymphoid cells in allergic disease. Curr Immunol Rev. 2013;9(4):214-221.

46. Asano K, et al. Intestinal CD169(+) macrophages initiate mucosal inflammation by secreting CCL 8 that recruits inflammatory monocytes. Nat Commun. 2015;6:7802.

47. Fallon PG, et al. IL-4 induces characteristic Th2 responses even in the combined absence of IL-5, IL-9, and IL-13. Immunity. 2002;17(1):7-17.

48. Moon TC, et al. Expression of DP2 (CRTh2), a prostaglandin D(2) receptor, in human mast cells. PLoS One. 2014;9(9):e108595.

49. McIvor RA. Emerging therapeutic options for the treatment of patients with symptomatic asthma. Ann Allergy Asthma Immunol. 2015;115(4):265-271 e5

50. Reif K, Cyster JG. RGS molecule expression in murine B lymphocytes and ability to down-regulate chemotaxis to lymphoid chemokines. J Immunol. 2000;164(9):4720-4729.

51. Sun Y, et al. Inhibition of the kinase ITK in a mouse model of asthma reduces cell death and fails to inhibit the inflammatory response. Sci Signal. 2015;8(405):ra122. 